\title{
Gravitational Dispersion Forces and Gravity Quantization
}

\author{
Fabrizio Pinto (D)
}

check for

updates

Citation: Pinto, F. Gravitational Dispersion Forces and Gravity Quantization. Symmetry 2021, 13, 40. https://doi.org/10.3390/sym13010040

Received: 30 November 2020 Accepted: 22 December 2020 Published: 29 December 2020

Publisher's Note: MDPI stays neutral with regard to jurisdictional clai$\mathrm{ms}$ in published maps and institutional affiliations.

Copyright: $\odot 2020$ by the author. Licensee MDPI, Basel, Switzerland. This article is an open access article distributed under the terms and conditions of the Creative Commons Attribution (CC BY) license (https:// creativecommons.org/licenses/by/ $4.0 /)$.
Ekospace Center and Department of Aerospace Engineering, Faculty of Engineering, Izmir University of Economics, Teleferik Mahallesi, Sakarya Cd. No:156, 35330 Balçova, İzmir, Turkey; fabrizio.pinto@ieu.edu.tr

\begin{abstract}
The parallel development of the theories of electrodynamical and gravitational dispersion forces reveals important differences. The former arose earlier than the formulation of quantum electrodynamics so that expressions for the unretarded, van der Waals forces were obtained by treating the field as classical. Even after the derivation of quantum electrodynamics, semiclassical considerations continued to play a critical role in the interpretation of the full results, including in the retarded regime. On the other hand, recent predictions about the existence of gravitational dispersion forces were obtained without any consideration that the gravitational field might be fundamentally classical. This is an interesting contrast, as several semiclassical theories of electrodynamical dispersion forces exist although the electromagnetic field is well known to be quantized, whereas no semiclassical theory of gravitational dispersion forces was ever developed although a full quantum theory of gravity is lacking. In the first part of this paper, we explore this evolutionary process from a historical point of view, stressing that the existence of a Casimir effect is insufficient to demonstrate that a field is quantized. In the second part of the paper, we show that the recently published results about gravitational dispersion forces can be obtained without quantizing the gravitational field. This is done first in the unretarded regime by means of Margenau's treatment of multipole dispersion forces, also obtaining mixed potentials. These results are extended to the retarded regime by generalizing to the gravitational field the approach originally proposed by McLachlan. The paper closes with a discussion of experimental challenges and philosophical implications connected to gravitational dispersion forces.
\end{abstract}

Keywords: dispersion potentials; phenomenology of quantum gravity; novel experimental methods; epistemology; semiclassical methods

\section{Introduction}

The Casimir effect is quite typically described as "an empirically verified quantum mechanical phenomenon involving an attractive force between two parallel uncharged mirrors in vacuum that exists even at zero temperature" [1]. The core of Casimir's original idea [2], which, with some variation of detail [3], he consistently traced to an early conversation with Bohr, is that a non-zero energy exists even in a vacuum. This is because the Uncertainty Principle of quantum mechanics prevents all components of the electric and magnetic fields to vanish identically at the same position in spacetime, so that contributions to the total energy density from electromagnetic fluctuations are expected [4]. Such energy is referred to as the zero-point energy (Nullpunktsenergie) - a concept that first emerged from Planck's "second theory" of the blackbody radiation in 1912 [5], then was famously considered in that context by Einstein and Stern [6] and by Bohr himself [7], and was finally extended to the electromagnetic field by Nernst [8] (for analyses of Nernst's work on zero-point energy see in [9-12]). The zero-point-energy exists also if two parallel plane reflectors separated by a gap of width $s$ are introduced. However, the discontinuities at the boundaries cause the energy density to change as a function of the gap width. A relatively simple calculation quickly shows that this leads to an attractive force, more correctly a pressure, proportional to $-\hbar c / s^{4}$, between the two plates [13]), where $\hbar$ and $c$ are Planck's 
constant and the speed of light in vacuum, respectively. This is now referred to as the Casimir force.

As quantum mechanics plays a key role in these interpretations, scientists and philosophers alike are presented with experimental confirmation of the Casimir force as evidence of the quantum nature of the associated field, in this case, the electromagnetic field. Such a point of view extends, more in general, to all electrodynamical dispersion forces, that is, forces due to the frequency-dependent electromagnetic polarizability of the interacting systems, whether they be macroscopic or microscopic. Despite the above matter-of-fact description [1] quite representative of the literature in the field, a robust debate regarding the logical relationship between electrodynamical Casimir forces and the principles of quantum mechanics has long been part of the research landscape.

In recent years, the subject of dispersion forces between gravitationally polarizable objects driven by spacetime fluctuations has attracted steadily increasing attention. Despite close field theory methodological analogies, however, the developmental trajectory of this research subfield has notably differed from that dealing with electrodynamical dispersion forces. Such a different path has unfolded in the nearly total absence of a parallel debate, thus leading to unquestioned claims that the detection of gravitational dispersion forces would conclusively prove the quantum nature of gravitation, claims we wish to discuss in this paper.

The plan for our analysis is as follows. In the following section, we review from a historical perspective the vigorous debate about the relationship between quantum atomic theory, quantum electrodynamics, and the existence of dispersion forces in both the unretarded and retarded regimes. In particular, we recall heuristic approaches and semiclassical theories leading to results indistinguishable from those obtained from the full quantum electrodynamical treatment. As an illustration that these descriptions are far from fruitless speculation, we mention and reference applications demonstrated to engineer dispersion forces in classical fields for specific technological purposes. In the following section (Section 3), we consider, again historically, developments leading to proposals about the existence of dispersion forces due to the gravitational field. The emphasis is on highlighting the fact that consideration of possible gravitational dispersion forces has occurred in the complete absence of a debate as to whether gravitational field quantization is necessary as a prerequisite for the existence of such forces, unlike what occurred in the electrodynamical case.

Readers not interested in these arguments may want to skip directly to our computational illustrations that gravitational dispersion forces can exist in the presence of classical gravitation with quantized atoms. As a first step (Section 4), we recall London's treatment of dipole's electrodynamical forces (Section 4.1) and Margenau's extension of such a formulation to multipole dispersion forces in the unretarded regime (Section 4.2). Those expressions are then reformulated in the classical gravitational case and it is shown that the recently published results obtained in linearized quantum gravity are immediately recovered (Section 5). New relationships, first suggested by Spruch on dimensional grounds, are also explicitly derived for the mixed quadrupole-quadrupole and dipolequadrupole electric gravitational potentials, which are tens of orders of magnitude larger than purely gravitational potentials (Section 8). The conclusions from this initial derivation are further strengthened by extending McLachlan's theory of dispersion forces to the gravitational case. For his purpose, we first recall McLachlan's theory (Section 6), in which the electromagnetic field is not quantized, and we show that it leads to the van der Waals and Casimir-Polder expressions in the unretarded and retarded regimes, respectively (Section 6.1). Then, restricting our treatment to the 1D case for succinctness, we consider the extension of McLachlan's approach to electric quadrupole dispersion forces (Section 6.2). The formulation of the same theory in the gravitational case is straightforward, as sketched out in Section 6.3.

We repeatedly stress throughout the paper that our efforts are not intended to support speculation that the electromagnetic field is not quantized. Instead, our starting point is that 
the conclusion that the electromagnetic field must be quantized could not be drawn based solely on the detection of dispersion forces. In fact, the existence of dispersion forces can be accommodated by quantizing the internal degrees of freedom of atoms interacting via classical electromagnetic fields. Therefore, the aim of this paper is to show that, analogously, any successful detection of gravitational dispersion forces could not be used to conclude that the gravitational field must be quantized. Quantized atoms interacting through a classical (general relativistic) gravitational field exhibit gravitational dispersion forces indistinguishable from those derived from linearized quantum gravity.

The challenging goal of detecting the gravitational equivalent of electrodynamical van der Waals, Casimir, and Casimir-Polder forces in the laboratory has been the subject of a few proposals, which are summarized in Section 7. The paper closes with a brief mention of connections between the main topic and two related subjects. On the one hand, the role played by dispersion forces within the philosophical idea of atomism is recalled, particularly as it regards the meaning of "void" in the presence of fluctuations of spacetime. On the other hand, some comments are provided about possible future technological applications of gravitational dispersion forces.

\section{The Quantum Structure of the Atom, Quantum Electrodynamics, and Dispersion Forces}

Historically, the first treatment of van der Waals forces between two hydrogen-like atoms was attempted by Wang [14] and later correctly completed by Eisenschitz and London [15] —indeed on the basis of the then newly developed principles of non-relativistic quantum mechanics. Such early studies were carried out contemporaneously with, but independently of, efforts to assemble the framework needed to describe the electromagnetic field in quantum mechanical language, the theory now referred to as quantum electrodynamics (QED) [16]. As lucidly clarified by London [17], who introduced the term "dispersion" in this context, the van der Waals force is explained in non-relativistic quantum theory as due to the existence of the zero-point-energy of perturbed harmonic oscillators, which schematically represent the interacting atoms. On the other hand, the electromagnetic field is assumed to be classical. Further developments, such as the calculation of atomic multipole effects, were built upon this same non-relativistic approach $[18,19]$.

Within just a few years, experimental data on lyophobic colloids [20] clearly demonstrated that the decay of van der Waals potentials at large particle separations is more rapid than predicted by the London treatment. Overbeek provided a suggestive, albeit only intuitive, explanation based on the effect of the finiteness of the speed of light, which, at large interparticle distances, would cause interacting classical resonators to vibrate outof-phase thus degrading their mutual attraction [21,22]. This concept brought to the fore the critical importance of retardation and led Casimir and Polder to attacking the problem of interatomic forces by means of the early tools of QED [23,24]. As recalled by Casimir, he followed up on this result by considering the attraction of two perfectly conducting neutral plates separated by a gap in terms of zero-point-energy of the electromagnetic field, so that "The problem in quantum electrodynamics is then reduced to a problem in classical electrodynamics" [25]. This approach, which Casimir humbly but entertainingly referred to as "Poor Man's QED" [26,27], led to his important discovery [2].

One important attribute of the "two parallel uncharged mirrors" to complete the above initial definition of the Casimir effect is that they are assumed to be perfect reflectorsclearly an extreme idealization. The strategy available to deal with intermolecular forces in real materials had been highlighted at least as early as in Einstein's very first published paper, in which he had been "... guided by the analogy with gravitational forces" ("Ich liess mich dabei von der Analogie der Gravitationskräfte leiten" [28]. Obviously, Einstein, writing even before the special relativity theory, is referring to Newtonian gravitation, which he would finally revise with his general relativity theory, in which gravitation is not trivially additive) [29]. Therefore, the van der Waals force between one atom and a slab or between two slabs was calculated by augmenting the theory by the ad hoc assumption of dispersion force additivity and by carrying out pairwise force summations in the continuum 
approximation [20,30-32]. However, it became quickly apparent that, unlike the case of Newtonian gravitation, additivity must actually be considered with suspicion. For instance, Axilrod and Teller intriguingly proved that each of three isolated atoms interacting via the unretarded van der Waals-London force not only do not experience forces trivially given by the pairwise sum, but can even mutually repel simply depending on their specific geometric arrangement [33].

Both the problem of retardation and of additivity in real materials, regardless of scale or distance and including absorption, were eventually addressed by Lifshitz through the introduction of a random electromagnetic field into the Maxwell equations [34,35]. Although the status of the Lifshitz theory in relationship to the fundamental principles of quantum mechanics has raised long-standing questions, as shown in the references above, the quantitative success of the resulting equations for the dispersion force between polarizable objects in explaining experimental data from a widespread variety of systems has been remarkable [36-39].

It is crucial to what follows that London's calculation of the near-range van der Waals force assumes a true vacuum, that is, "... a state with all physical properties equal to zero" [40], within which to consider the classical electrostatic interaction of two instantaneous dipoles described by non-relativistic quantum mechanics. On the other hand, the treatment of the long-range potential presented by Casimir and Polder is explicitly rooted in the concept of the zero-point-energy of the quantum vacuum [41]. This seems to imply, as is often stated, that the existence of retarded interactions cannot be explained outside the framework of quantum field theory. However, this is not the case. Indeed, somewhat in the path Overbeek had envisioned, theories leading to the correct retarded potentials have been developed "in spite of having used the nonretarded Schrödinger equation" [42] to treat the interacting atoms but retaining the electromagnetic field as classical. As the quantum nature of the electromagnetic field appears beyond doubt on independent considerations, such semiclassical endeavors are at times presented self-deprecatingly, in a manner mindful of, or perhaps in order to pre-empty, Osiander's opinion that, for the sole purpose of saving the phenomena, "... these hypotheses need not be true nor even probable ..." [43] ("Neque enim necesse est, eas hypotheses esse veras, imo ne verisimiles quidem ..." [44]). For instance, in a short pedagogical article aimed at illustrating the basic idea of the Casimir effect, Kleppner instead discusses, "delightfully" [45], a onedimensional version [46] of London's unretarded model of interacting harmonic oscillators [17]. The obvious extension of that approach to include full retardation-the actual Casimir effect-is disposed of as to "flog the argument beyond the point of diminishing returns" [47].

One recurring apologetic argument for all such efforts is that pedagogy can take advantage of "...the considerable simplification in the mathematical aspects of the framework over the approach based on quantum electrodynamics" [48]. A slightly more ambitious perspective holds that "The various interpretations of the dispersion effect are aimed at minimizing the quantum theoretical effort" but "It is one of the objectives of the quantum electrodynamics procedure to check the errors and limits of these semiclassical approaches" [42].

Along these lines, additional motivation was provided by Casimir and Polder themselves in their early speculation that "... it might be possible to derive these expressions, perhaps apart from the numerical factors, by more elementary considerations. This would be desirable since it would also give a more physical background to our result, a result which in our opinion is rather remarkable" [24]. This goal was adopted by McLachlan, who explained: "In one sense the theory of molecular attractions is now complete, but there is still a need for an elementary discussion which gives better physical insight. The aim of this paper is to find the dispersion force between two molecules by a new method which uses elementary quantum mechanics and assumes almost no knowledge of quantum field theory" [49]. Indeed, Margenau, commenting in the aftermath of such work, stated that 
"The approach just considered, for which we are indebted to McLachlan, is essentially classical, the only quantum mechanics occurring in the definition of the polarizability" [50].

At the far end of the spectrum, one finds efforts not just aimed at recovering known results with a less onerous mathematical apparatus for reasons of succinctness or insight but claiming to do so, regardless of complexity, in order to show that the nature of the electromagnetic field is not fundamentally quantum mechanical. In this view, referred to as "stochastic" or "random" electrodynamics (SED), there actually exists a fluctuating zero-point electromagnetic field but such an object is completely classical and it appears as a solution of the homogeneous wave equation for the potential vector [11]. As pointed out by Marshall [51,52] and Boyer [53], the theory is Lorentz-invariant, and it has been shown not only to recover the major expressions from "standard" dispersion force theory but also-stimulated by a question from none other than Casimir himself-to lead to the earliest prediction of a repulsive Casimir force between a perfectly electrically conducting and a perfectly magnetically permeable slab [54] (see also in [55]).

The fact that this semiclassical approch and "standard" QED calculations lead to the same expressions was stressed by Spruch, who observed: "Why vacuum fluctuation arguments worked in the past in the problems to which they were applied is, to our knowledge, not completely understood, but the simplicity of the approach gives it considerable appeal, as a means of providing physical insight into known results and as a means of suggesting new results" [56]. Indeed, Spruch adds that "it is not necessary to go to the trouble for almost all of the work has been done, and we simply note down an extension of a version given by Boyer". Interestingly, the result used therein had been obtained by Boyer by pointing out that "we may regard the fields equally well as classical fields subject to a random walk or as quantum fields" [53]. Several years later, Spruch presented these arguments in a remarkable pedagogical article written for Physics Today to illustrate that "... the essential idea is to proceed entirely classically... until that last step ... in which we quantize the energy of the modes of vibration of the electromagnetic field" [57]. This approach yields a completely classical expression for the dispersion energy for any arbitrary specific energy density, and to the intriguing comment that such is "... a result Maxwell could have derived, and perhaps did". (The reasons that this "counterfactual history" statement cannot be considered realistic were explored in [22]) Finally, Spruch states that "[T] he quantum version follows immediately on making $\mathbf{E}_{0}$ the field of vacuum fluctuations ... in that case the energy in a mode of the field is simply $\ldots \frac{1}{2} \hbar \omega \ldots "$. We must notice, however, that whereas that "last step" is attributed the actual meaning of field quantization by Spruch, within the SED framework the fields remain classical and Planck's constant " ... $\hbar$ is regarded as nothing more than a number chosen to obtain consistency of the predictions of the theory with experiment" [11].

Predictably, a classical electromagnetic zero-point field is perceived as a highly controversial proposal [3] —indeed Boyer noted that "some readers ... are distressed, even indignant at the idea ..." [58] — and Milonni concluded that "In spite of the successes of SED, it cannot at this time be considered a serious alternative to QED" [11]. Such peremptory rejection, supported by the argument that, for instance, "no classical theory of the electromagnetic field can account for such experimentally observed phenomena as the photon polarization correlations in a cascade radiative decay of atomic states" [11], has, however, not resulted in the disappearance of the SED approach. From the theoretical standpoint, this is continues to be presented as being due, in a typical literature example, to "computational advantages" [59], couched in Osiander's language of profuse apology despite excellent agreement with experimental data, confessing that the purpose "... is not to propound some rival to QED" [59].

Even more importantly, continued pursuit of the SED standpoint eventually inspired novel experimental physics and engineering applications of the Casimir effect. One development was motivated by a description of dispersion forces in terms of the net radiation pressure of all modes of the zero-point-field first suggested by Casimir in his landmark publication [2], quoted verbatim much later by Debye [9] (see in [60], Note 5), and fully ex- 
plored by Gonzáles [61] and, finally, by Milonni [11,62]. Despite some commentary critical of this famously lucid and oft-cited interpretation, delivered by Barton in a section tellingly entitled "Exorcism" [63], the prediction of the existence of forces between macroscopic boundaries does not appear to demand a quantum origin of the field, which can thus be classical. Therefore, both Boyer's SED papers and Milonni's more recent radiation pressure formulation are consistently cited as cornerstones in theses and articles leading to the successful detection of the acoustic Casimir effect [64-66], that is, "the force between two plates in a homogeneous, isotropic, acoustic field" [67]. The development of this research subfield led to a deeper understanding of the connection between the detailed features of the random vibration spectrum with the Casimir force as a function of the gap width between the two plates. Whereas, as we stated above, in the electromagnetic case such spectrum is uniquely determined by the requirements of Lorentz invariance, in the acoustic case it can be arbitrarily set by the experimenter.

The outcome of these studies was the mathematical proof that a narrow band random noise spectrum may result in large oscillations of the force with distance, later independently re-discovered by Ford in the electromagnetic case [68], which finally led to the first experimental detection of repulsive acoustic Casimir forces [66,67]. The remarkable possibility to tailor such interactions in intensity and sign suggested that engineering the noise spectrum might lead to novel applications in micromanipulation and particle levitation. More recently, this interesting result has led to the theoretical suggestion [69] that high-frequency noise fields might represent a tool to control stiction, the phenomenon responsible for nonlinearities and a failure mode in microelectromechanical systems (MEMS) [70]. The Casimir effect with classical random fields - referred to by some as the "analog" Casimir effectwas further demonstrated by measuring the force between two vertically plates partially submerged in a dish containing a liquid (This interesting experiment has been presented as indirect support of the veracity of historical reports of a "maritime" Casimir effect, that is, the force attraction of two side-by-side ships in a rough sea [71]. Despite the correctness of the experiments reported in [72], the present author has shown [73] that there exists no basis to believe that the drawing shown in [71] suggests that "It was believed in the days of the clipper ships that ... two vessels at close distance [in a strong swell] will attract each other". In fact, as also remarked by a reader in a letter to the Editor [74], the traditional belief was the opposite, that is, that ships in a completely flat sea attract. However, the captions of the two figures in the historical source, one showing rough seas and other one flat calm, were inexplicably swapped by the author of [71]) excited by a shake table [72].

As a final example of productivity of the SED point of view, again represented by citation of Boyer's papers as the logical starting point, we mention the very recent experiments aimed at engineering dispersion forces by means of arbitrary fluctuating electromagnetic fields in optical nanoparticle manipulation applications [75]. This remarkable work has generated renewed impetus in long standing efforts to shape dispersion forces by radiation [76] resulting in applications in bacterial screening [77] and in further progress in "gravitational-like" interactions [78-80] and "mock gravity" [81].

We conclude these introductory remarks by quoting leading research protagonists implicitly or explicitly exposing the need to accept the limitations presented by using forces between polarizable particles as a tool to ascertain the ultimate nature of the fields. For instance, the discoverers of "optical binding" offered the following commentary applicable to the issue at hand.

"Finally we note that many readers of this journal view forces between elementary particles of nature as originating from the exchange of virtual quanta of fields to which they are coupled. The induced interaction discussed in this paper fits nicely into that scheme, but with real quanta being exchanged. We wonder whether other particles and fields may be substituted for our dipoles and light, yielding analogous effects in other domains of physics" [82].

An obvious illustration of this argument is the view of the Casimir effect as due to radiation pressure of a zero-point-field photons gas-an interpretation that cannot differentiate 
between real or virtual photons. Speaking more broadly, DeWitt has remarked that "There must be nearly two dozen ways of calculating the Casimir effect" [83]. Indeed, approaches as diverse as QED and SED lead to the same expressions for dispersion forces in various regimes, thus suggesting that the existence of dispersion forces is not a valid tool to determine whether a field is quantized. This was explicitly noted by the researcher who designed the first modern experiment to accurately test the predictions of the Lifshitz theory [84], S. K. Lamoreaux, who stated: “There are physical phenomena that truly require a quantization of the electromagnetic field for their explanation; the Casimir force is not among these phenomena, because the predictions based on different points of view are identical" [85].

As is tradition, the present author also proffers his own apology of quantum electrodynamics, which has in fact never been falsified and is consistently hailed, with reason, as possibly the most successful physical theory ever devised by the human mind [86]. However, special care must be used as we attempt to employ dispersion forces to draw conclusions about the nature of "other particles and fields". In this sense, it is appropriate to extensively quote some words by Richard Feynman in his Nobel Prize acceptance speech [87]:

"Physical reasoning does help some people to generate suggestions as to how the unknown may be related to the known. Theories of the known, which are described by different physical ideas may be equivalent in all their predictions and are hence scientifically indistinguishable. However, they are not psychologically identical when trying to move from that base into the unknown. For different views suggest different kinds of modifications which might be made and hence are not equivalent in the hypotheses one generates from them in ones attempt to understand what is not yet understood. I, therefore, think that a good theoretical physicist today might find it useful to have a wide range of physical viewpoints and mathematical expressions of the same theory (for example, of quantum electrodynamics) ..."

It may appear surprising to see an extraordinarily objective physicist as Feynman ponder about such concepts as "psychologically identical" ideas-a criticism he seems to pre-empty at the onset by stating that he just wants "to make the lecture more entertaining". However, considering that Julian Schwinger, seated in that same audience that day, would later refer to the Casimir effect as "One of the least intuitive consequences of quantum electrodynamics" [40], Feynman's warning must be taken very seriously as we move from the known, QED, to the unknown, the ultimate structure of spacetime.

\section{Gravitational Dispersion Forces}

It is a puzzling fact in the history of this subfield that no treatment of gravitational dispersion forces was, to the best knowledge of this author, ever published till very recently although, for instance, the non-relativistic regime does not require a treatment remarkably more complex than that developed by London. This silence was not due to a lack of sophisticated theoretical tools. The calculation of classical (i.e., non-quantum) corrections to the gravitational force upon a charge distribution in various gravitational fields was pursued intermittently, often without awareness of past results [88], as the first attempt by Enrico Fermi to study electrostatics within the then-novel theory of general relativity while at at the Scuola Normale Superiore at Pisa [89].

Even as QED was being developed, the problem of quantizing linearized gravity had also emerged [90-92], most prominently, in the pioneering works of Rosenfeld [93] and Bronstein [94]. The results by the latter are remarkable from our perspective even in the present day because, as pointed out by the editors [95] of his recently reprinted work [96], Bronstein even addressed the presence of a gravitational zero-point-energy and implemented the proper operator ordering [11] needed to avoid it (This now famous paper was Bronstein's PhD dissertation, presented in 1935. He was arrested in August 1937 during the Great Purge, then tried, sentenced, and shot on the same day in a Leningrad 
prison on 18 February 1938 [91,97]). His results, achieved at a remarkably early stage in the exploration of the challenges of a theory of quantum gravity and "Published in German in a journal inaccessible today" [95], remained relatively unknown. Indeed, as late as 1992, Gorelik still found the need to openly contradict Steven Weinberg's taking "liberties with history" [90] for rashly awarding to Planck [98] priority in identifying the "inconsistency between quantum mechanics and general relativity", an achievement which Gorelik attributed to Bronstein.

In the several decades that followed, corrections to the Coulomb and post-Newtonian potentials, as well as mixed potentials, were derived both within classical general relativity and quantum field theory frameworks [99-104] (the contemporary state-of-the-art is reviewed in [105-107]). The relatively late development of the gravitational potential corrections was explicitly acknowledged by Feinberg, Sucher, and Au as late as 1989: "Two-graviton exchange has also been studied in some approximations ... but, a general expression for the potential arising from this exchange, similar to that ... for two-photon exchange between charges has, to our knowledge, not been derived" [108]. Specifically regarding corrections to the Coulomb potential, the same authors observe "It is interesting to note that the $r^{-2}$ term is independent of Planck's constant. Indeed, this term can be obtained in a purely classical treatment of electrodynamics ... This is not the case for the $r^{-3}$ term". It is a further demonstration of the tortuous history of the subject that the classical $r^{-2}$ term had already been exhibited by Berends and Gastmans in 1976 [100] but was recognized by them, "in proof", as having being published by Cécile and Bryce DeWitt as early as 1964 [99].

As far as the quantum corrections to the gravitational as well as the mixed potentials $\left(\propto r^{-3}\right)$, remarkably early comments are found in the aforementioned paper by Spruch [57] and reiterated in a later review chapter on Casimir forces, also of a pedagogical nature [109]. For instance, after having obtained the order of magnitude of the correction to the Coulomb potential, $V_{\mathrm{ee}} \sim \hbar e^{4} / c^{3} m^{2} r^{3}$, Spruch instructs the reader: "To obtain the gravitational analog of the electron-electron interaction, replace both factors of $e^{2}$ by $\mathrm{Gm}^{2}$; to obtain the gravitational-electromagnetic interference term in the interaction, replace only one factor of $e^{2}$ with $G m^{2}$ ". As noted later, as this mixed contribution is linear in $G$, it is "much larger and more interesting" (see in [57], Equation (6), box on p. 42, and p. 43; see also in [109], p. 28). However, having come so close to the subject of dispersion forces in gravitationally polarizable systems, Spruch walks away from it with the justification that "...there is no gravitationally neutral system and therefore no gravitational analog of the electrically neutral atom ...".

An element crucial to the focus of the present paper was added to the debate by J. P. Dowling in a letter written in response to Spruch's article in Physics Today [110]. Dowling reminded readers that, as we repeatedly mentioned above, there exist other points of view than explaining the existence of retarded dispersion forces by coupling of the interacting systems with the electromagnetic zero-point fluctuations, such as the Lifshitz theory [34] and the source theory by Schwinger, DeRaad, and Milton [40]. To these, he added yet another framework, that is, the "self-field approach" in which no second quantization is carried out, as proposed by Barut [111,112] and pursued by him and his collaborators, including J. F. Van Huele [113] and Dowling himself [114,115]. In the apologetic tone typical of authors straying from the standard theory, Barut and Dowling responded by citing the facts: "If a semiclassical theory is defined as a theory which is not second quantized, then self-field QED has been quite a successful semiclassical theory (at least to order $\alpha$ ) in accounting for quite an array of phenomena thought to require at least the second quantization of the radiation field for their explanation" [115]. Among such phenomena, were also the "long-range Casimir-Polder van der Waals forces near boundaries" [114]. The obvious consequence of such observations is, once again, that if the existence of electrodynamical dispersion forces does not demand second quantization but can be correctly described within a semiclassical theory, neither does the existence of gravitational dispersion forces. 
In the last forty years, the subject of dispersion forces involving the gravitationally field has been slowly, and rather haphazardly, rediscovered. Among earliest studies in this phase were calculations of the gravitational Casimir energy in very specific contexts of cosmology and field theory [116-118]. As late as 1993, Spruch had dismissed the problem stating: "We have been concerned almost exclusively with electromagnetic effects but it is amusing to consider gravitational effects, and it is trivial to do so" [109]. Apparently unaware of Spruch's assessment, Panella and Widom wrote, shortly thereafter, that "... to the best of our knowledge, the study of long range gravitational interactions between massive bodies (e.g., calculation of gravitational retarded static potentials) has not yet been undertaken". In their article, these authors presented possibly the earliest detailed calculation of gravitational dispersion forces- "a new finite effect of linearized quantum gravity namely, the retarded (Casimir) potential of a test mass interacting with a condensed matter system" [119]. Disappointingly, their result—-the retarded gravitational potential between a point mass and an extended mass distribution found by an approach that parallels that used to analyze the analogous electrostatic problem [120] - remained virtually uncited till 2017.

Approximately two decades after Panella and Widom, the problem reemerged from two independent directions. On the macroscopic scale, the original motivation was provided by theoretical speculation-tempered by extreme skepticism in the absence of any experimental confirmation - that superconductors might act as nearly-ideal high frequency gravitational wave reflectors, thus opening the door to a new field of optics [121-124]. Following a suggestion by Bouwmeester, reported by Minter et al. [125], the possibility of gravitational wave reflection led to a treatment of the gravitational Casimir effect (Importantly, Casimir's approach was later explicitly mentioned by Sakharov (reprinted in [126]) in his theory of "induced gravity" [127]. Although effects connected to fluctuations in curved spacetime are sometimes referred to as a "gravitational Casimir effect" [128], in this paper, we consider gravitation as a fundamental, and not an emergent, interaction) analogous to that of the Lifshitz theory in QED $[129,130]$. Very shortly thereafter, and without any reference to the results by Quach $[129,130]$ in the macroscopic regime, the computation of the gravitational van der Waals and Casimir-Polder potentials appeared, carried out by means of quantum field theoretical methods by Ford, Hertzberg, and Karouby [131], confirmed a few months later by $\mathrm{Wu}, \mathrm{Hu}$, and $\mathrm{Yu}$ [132]; $\mathrm{Hu}$ and $\mathrm{Yu}$ [133]; and Holstein [134].

On the one hand, the macroscopic scale results $[129,130]$ have stimulated a justifiably lively debate as to whether a hypothetical detection of such a gravitational Casimir effect would in fact represent a conclusive confirmation of the quantum nature of spacetime $[22,60,135-138]$. On the other hand, the microscopic scale calculations have been accompanied by a unanimous consensus on the part of those who carried them out that any experimental confirmation of those predictions would reveal a signature of the quantum nature of the gravitational field. Consider the following examples of matter-of-fact commentary provided regarding the first black hole merger detection, announced within the same time frame as the above results [139], and gravitational dispersion forces. Holstein forcefully stated that "The recent observation at LIGO of gravitational radiation has verified the existence of gravitons and has emphasized the importance of studying processes involving their interactions" [134]. Ford and collaborators sweepingly concluded that their own work "... is a rare, precise, and definite prediction of quantum gravity, independent of the details of its UV completion ... (analogous to the electromagnetic Casimir-Polder and London-van der Waals forces) ..." [131]. Along these lines, $\mathrm{Wu}, \mathrm{Hu}$, and $\mathrm{Yu}$ introduced their calculations stating that "Although a full theory of quantum gravity is absent, one can still use linearized quantum gravity to find quantum gravitational corrections to classical physics which an ultimate quantum gravity theory must produce at low energies" [132]. Finally, $\mathrm{Hu}$ and $\mathrm{Yu}$ commented that "One naturally expects that, if gravity has a quantum nature, it should also generate Casimir-like forces" [133].

As must appear obvious in light of the facts we recalled so far, all such strong claims deserve careful analysis to avoid logical pitfalls. For instance, as we have repeatedly seen, 
Casimir forces are also predicted by theories without second quantization and even with entirely classical fields $[140,141]$. Therefore, a non-quantum gravitational field should still very much be expected to generate Casimir forces. Furthermore, in electrodynamics, one would find it obviously untenable to state that "the observation of light verifies the existence of photons". Indeed, Dev and Mazumdar voiced the opinion that "The LIGO detection as such does not confirm whether the observed gravitational wave is classical or quantum" [142].

From the methodological standpoint, as the present author has previously discussed [60], the adoption by Ross and Moreau of the SED mathematical tools developed by Boyer to carry out an analogous study of "stochastic gravity" [143] is neither less rigorous nor less promising than far more popular linear quantum gravitational methods. For instance, in addition to providing a useful framework to treat gravitation approaching the Planck scale, the possibility must be examined that contamination of gravitational dispersion force measurements [60] might occur from an as yet undetected classical gravitational stochastic field background of cosmological and astrophysical origins [144-146].

In summary, the historical evidence has shown that the developmental trajectory of the quantum gravity research subfield intriguingly differed from that of QED. Whereas at least half-a-century of extensive analysis in the electrodynamical domain established that "... the Casimir effect reveals nothing conclusive about the nature of the vacuum" [147], claims that "If gravity truly has a quantum nature, then gravitational waves should also generate Casimir-like forces" [135] were left largely unchallenged.

In the remainder of this paper, we discuss some examples of calculation of gravitational dispersion forces carried out assuming that the gravitational field is classical.

\section{Unretarded Higher Multipole Electrodynamical van der Waals Forces}

The computational strategy adopted below is part of the ongoing program by this author to bring computer algebra system (CAS) technology to bear in attacking prohibitively complex Casimir force problems [22,148]. This approach is particularly effective when generalizing computations to systems in which simplifying symmetries may be lost, as in the case of electrodynamical dispersion forces in the presence of arbitrary gravitational fields [88], to avoid errors due to very extensive algebraic calculations or to identify errors in the published literature, as well as for pedagogical reasons. In order to demonstrate the algorithm, first we verify several well known expressions for the generalized electric multipole van der Waals potential by means of the Mathematica ${ }^{\mathrm{TM}}$ system (v. 11.3.0.0). Second, we proceed by adapting this approach to compute the gravitational van der Waals potential expressions. Finally, we report on the explicit calculation of the mixed electric-gravitational potentials in the unretarded regime.

\subsection{Margenau's Algorithm: London Potential}

The interatomic London potential $[15,17]$ will be calculated by the procedure developed by Margenau [18], who first computed the quadrupole contributions to the van der Waals interatomic forces on Frenkel's suggestion. This is summarized here for convenience. Let us consider the Coulomb potential (Gaussian units), $V(\mathbf{r})=e /\left|\mathbf{r}-\mathbf{r}_{\mathbf{0}}\right|$, at $\mathbf{r}$ (components $\left.x^{i}\right)$ due to a proton of charge $e=+|e|$ placed at $\mathbf{r}_{0}$ (components $x_{0}^{i}$ ). The total potential $V_{\mathrm{pe}}$ at $\mathbf{r}$ due to this proton and to its atomic electron of charge $-e$, placed at a position $\mathbf{r}_{1}$ away from the proton is, to second order in the components $x_{1}^{i}=x^{i}-x_{0}^{i}$ (index summation convention is used) and eventually setting $\mathbf{r}_{\mathbf{0}}=\mathbf{0}$ :

$$
V_{\mathrm{pe}}(\mathbf{r})=\frac{e}{r}-\frac{e}{r}+e\left(x_{1}^{i} \frac{\partial}{\partial x^{i}}\right)\left(\frac{1}{r}\right)+\ldots,
$$

where $\partial / \partial x=-\partial / \partial x_{0}$. Let us now place at second atom with proton at $\mathbf{r}$ and electron at $\mathbf{r}_{2}$ from the proton. The classical dipole-dipole potential energy of this atom in the potential $V_{\text {pe }}(\mathbf{r})$ is, also to first order in $x_{2}^{k}$ : 


$$
W_{\mathrm{dd}}\left(\mathbf{r}_{1}, \mathbf{r}_{2}, \mathbf{r}\right)=e V_{\mathrm{pe}}(\mathbf{r})-e V_{\mathrm{pe}}(\mathbf{r})-e\left(x_{2}^{k} \frac{\partial}{\partial x^{k}}\right) V_{\mathrm{pe}}(\mathbf{r})+\cdots=-e^{2}\left(x_{1}^{i} x_{2}^{k} \frac{\partial}{\partial x^{i}} \frac{\partial}{\partial x^{k}}\right)\left(\frac{1}{r}\right) \ldots
$$

By implementing the above steps in Mathematica, and by choosing $\mathbf{r}=(R, 0,0)$, we find

$$
W_{\mathrm{dd}}\left(\mathbf{r}_{1}, \mathbf{r}_{2}, R\right)=-\frac{e^{2}}{R^{3}}\left(2 x_{1}^{1} x_{2}^{1}-x_{1}^{2} x_{2}^{2}-x_{1}^{3} x_{2}^{3}\right),
$$

which is the classical perturbing field used by London. As is well known [18,149], the calculation next proceeds to obtain a good approximation of the expression for the van der Waals force by evaluating the following six-dimensional integral,

$$
U_{\mathrm{vdW}}(R) \simeq-\frac{1}{2 E_{I}} \int_{V_{1}} \int_{V_{2}} \phi_{1,0,0}^{*}\left(\mathbf{r}_{\mathbf{1}}\right) \phi_{1,0,0}^{*}\left(\mathbf{r}_{\mathbf{2}}\right) W_{\mathrm{dd}}^{2}\left(\mathbf{r}_{\mathbf{1}}, \mathbf{r}_{\mathbf{2}}, R\right) \phi_{1,0,0}\left(\mathbf{r}_{\mathbf{1}}\right) \phi_{1,0,0}\left(\mathbf{r}_{\mathbf{2}}\right) d \mathbf{r}_{\mathbf{1}} d \mathbf{r}_{\mathbf{2}} ，
$$

where $E_{I}$ is the atomic ionization energy $E_{I}=e^{2} / 2 a_{0}$ and $a_{0}=\hbar^{2} / m_{e} e^{2}$ is the Bohr radius and the normalized ground state wave function $\phi_{1,0,0}$ is:

$$
\phi_{1,0,0}(\mathbf{r})=\frac{e^{-r / a_{0}}}{\sqrt{a_{0}^{3} \pi}}
$$

The elementary process consists of considering the symmetry properties of the integrals of the 6 terms produced by squaring the classical energy at Equation (3) and recognizing that some vanish while all others are identical to one another. Here, instead, we proceed by brute force as all such integrals are elementary and can be computed by Mathematica in $\sim 1 \mathrm{~s}$ of CPU time on a typical laptop. With the above approximations, one finds the standard expressions:

$$
U_{\mathrm{vdW}}(R)=-\frac{3 a_{0}^{4} e^{4}}{E_{I} R^{6}}=-\frac{6 a_{0}^{5} e^{2}}{R^{6}} .
$$

This quantity can be expressed in terms of $\left.<1,0,0\left|r^{2}\right| 1,0,0\right\rangle=r_{1,0,0}^{2}=3 a_{0}^{2}$, so that we can finally write:

$$
U_{\mathrm{vdW}}(R)=-\frac{e^{4}\left(r_{1,0,0}^{2}\right)^{2}}{3 E_{I} R^{6}},
$$

which is the expression given by Margenau (see in [18], Equation (8) and in [50], p. 24). Further expressions of this result useful for comparison with the literature can be obtained by writing the square of the norm of the ground state dipole expectation value from the result above as $\left\|\mu_{1,0,0}\right\|^{2}=3 e^{2} a_{0}^{2}$, so that

$$
U_{\mathrm{vdW}}(R)=-\frac{\left(\left\|\mu_{1,0,0}\right\|^{2}\right)\left(\left\|\mu_{1,0,0}\right\|^{2}\right)}{3 E_{I} R^{6}},
$$

which agrees with the complete expression at Equation (1.1) of the work in [150]:

$$
U_{\mathrm{vdW}}(R)=-\frac{2}{3 R^{6}} \sum_{r, s} \frac{\left\|\mu_{0, r}(A)\right\|^{2}\left\|\mu_{0, s}(B)\right\|^{2}}{E_{r, 0}+E_{s, 0}},
$$

in the approximation in which one only retains the ground state contribution of two identical atoms, $A$ and $B$. Finally, let us write the electric dipole polarizability from the Kramers-Heisenberg formula [11,150,151]:

$$
\alpha_{\mathrm{E}}^{(1)}(\omega)=\frac{2}{3} \sum_{r} \frac{E_{r, 0}\left\|\mu_{0, r}\right\|^{2}}{E_{r, 0}^{2}-(\hbar \omega)^{2}} .
$$


In the static limit for $\omega \rightarrow 0$, again only considering the contribution of the ground state and by using the results above, we find the classical static dipolar polarizability, $\alpha_{0, \mathrm{E}}^{(1)}=4 a_{0}^{3}[11,152]$, and we obtain another standard expression:

$$
U_{\mathrm{vdW}}(R)=-\frac{3 E_{I}\left(\alpha_{0, \mathrm{E}}^{(1)}\right)^{2}}{4 R^{6}}
$$

\subsection{Margenau's Algorithm: Quadrupole van der Waals Forces}

The next objective to validate our approach is to generalize the above procedure to higher multipoles by first expanding $V_{\mathrm{pe}}(\mathbf{r})$ to second order at Equation (1):

$$
V_{\mathrm{pe}}(\mathbf{r})=e\left(x_{1}^{i} \frac{\partial}{\partial x^{i}}-\frac{1}{2} x_{1}^{i} x_{1}^{j} \frac{\partial}{\partial x^{i}} \frac{\partial}{\partial x^{j}}\right)\left(\frac{1}{r}\right)+\ldots
$$

Therefore, the electrostatic potential energy of the two atoms is

$$
\begin{aligned}
W_{\mathrm{dd}}\left(\mathbf{r}_{1}, \mathbf{r}_{2}, \mathbf{r}\right) & =-e\left(x_{2}^{k} \frac{\partial}{\partial x^{k}}+\frac{1}{2} x_{2}^{k} x_{2}^{l} \frac{\partial}{\partial x^{k}} \frac{\partial}{\partial x^{l}}\right) V_{\mathrm{pe}}(\mathbf{r})+\ldots \\
& =-e^{2}\left(x_{2}^{k} \frac{\partial}{\partial x^{k}}+\frac{1}{2} x_{2}^{k} x_{2}^{l} \frac{\partial}{\partial x^{k}} \frac{\partial}{\partial x^{l}}\right)\left(x_{1}^{i} \frac{\partial}{\partial x^{i}}-\frac{1}{2} x_{1}^{i} x_{1}^{j} \frac{\partial}{\partial x^{i}} \frac{\partial}{\partial x^{j}}\right)\left(\frac{1}{r}\right) .
\end{aligned}
$$

By implementing these expansions in Mathematica and again choosing $\mathbf{r}=(R, 0,0)$, we obtain the sum of three polynomials proportional to $1 / R^{3}, 1 / R^{4}$, and $1 / R^{5}$, where the first of course recovers the dipole-dipole potential energy seen above. Squaring this quantity to obtain $W_{\mathrm{dd}}\left(\mathbf{r}_{1}, \mathbf{r}_{2}, R\right)$ leads to a polynomial that Mathematica measures as having 229 terms. The 6-dimensional integration shown at Equation (4), carried out in $\approx 180 \mathrm{~s}$ of CPU time, yields the following van der Waals potential,

$$
U_{\mathrm{vdW}}(R)=-\frac{3 a_{0}^{4} e^{4}}{E_{I} R^{6}}-\frac{135 a_{0}^{6} e^{4}}{2 E_{I} R^{8}}-\frac{2835 a_{0}^{8} e^{4}}{4 E_{I} R^{10}}=-\frac{6 a_{0}^{5} e^{2}}{R^{6}}-\frac{135 a_{0}^{7} e^{2}}{R^{8}}-\frac{2835 a_{0}^{9} e^{2}}{2 R^{10}},
$$

coinciding with the results by Margenau [18,50] and Pauling [153] (In Equation (47.7) [154], the coefficient of the term in $1 / R^{10}$ is given as 1416 instead of 1417.5). Margenau also expresses this result [18] in terms of the quantity $\left\langle 1,0,0\left|r^{4}\right| 1,0,0\right\rangle=r_{1,0,0}^{4}=\frac{45}{2} a_{0}^{4}$ and of $r_{1,0,0}^{2}$ already found above:

$$
U_{\mathrm{vdW}}(R)=-\frac{e^{4}\left(r_{1,0,0}^{2}\right)^{2}}{3 E_{I} R^{6}}-\frac{e^{4}\left(r_{1,0,0}^{2} r_{1,0,0}^{4}\right)}{E_{I} R^{8}}-\frac{7 e^{4}\left(r_{1,0,0}^{4}\right)^{2}}{5 E_{I} R^{10}}
$$

Finally, let us express this result in terms of the dipole moment found above and of the traceless quadruple moment tensor defined as [150,155-157]

$$
Q_{i j}=-\frac{e}{2 !}\left[x^{i} x^{j}-\frac{1}{3} r^{2} \delta_{i j}\right]
$$

Computing the expectation values $<1,0,\left.0|| Q_{i j}\right|^{2} \mid 1,0,0>$ by means of Mathematica, the square of the Frobenius norm [158] of the ground state traceless quadruple moment tensor can be obtained:

$$
\|Q\|_{F}^{2} \equiv \sum_{i} \sum_{j}<1,0,0 \|\left. Q_{i j}\right|^{2} \mid 1,0,0>=\frac{15}{4} a_{0}^{4} e^{2} .
$$

Let us now use this result in the quadrupole-quadrupole, $1 / R^{10}$ term at Equation (14), indicated as $V_{22}(R)$ in [150]:

$$
V_{22}(R)=-\frac{2835 a_{0}^{8} e^{4}}{4 E_{I} R^{10}}=-\frac{504}{5 R^{10}} \frac{\left(\|Q\|_{F}^{2}\right)\left(\|Q\|_{F}^{2}\right)}{2 E_{I}},
$$


in agreement with Equation (1.3) of the work in [150]. By now using Equation (17) and the norm squared of the ground state dipole expectation, $\left\|\mu_{1,0,0}\right\|^{2}=3 e^{2} a_{0}^{2}$, we can rewrite the dipole-quadrupole, $1 / R^{8}$ term at Equation (14), indicated as $V_{12}(R)$ in [150], as

$$
V_{12}(R)=-\frac{135 a_{0}^{6} e^{4}}{2 E_{I} R^{8}}=-\frac{135}{R^{8}} \frac{1}{2 E_{I}} \frac{4}{15}\left(\|Q\|_{F}^{2}\right) \frac{1}{3} \|\left(\mu_{1,0,0} \|^{2}\right)=-\frac{12}{R^{8}} \frac{\left(\|Q\| \|_{F}^{2}\right)\left(\mu_{1,0,0} \|^{2}\right)}{2 E_{I}}
$$

which indicates the factor of 3 in front of the summation at Equation (1.2) of the work in [150] should be 12.

Finally, let us express these results in terms of the static dipole $\left(\alpha_{0, \mathrm{E}}^{(1)}=\frac{2}{3}\left\|\mu_{0, r}\right\|^{2} / E_{r, 0}\right)$ and static electric quadrupole polarizabilities, by using the standard expression for the latter (i.e., see Equation (6.5) in [159]), where $\mu_{0, r}$ and $E_{r, 0}$ are the transition moments and the transition energies, and again only considering the ground state contribution [150]:

$$
\alpha_{0, \mathrm{E}}^{(2)}=\frac{1}{5} \sum_{r} \frac{E_{r, 0}\|Q\|_{F}^{2}}{E_{r, 0}^{2}-(\hbar \omega)^{2}} \longrightarrow 2 \frac{\left(\|Q\|_{F}^{2}\right)}{E_{I}}
$$

In summary, we find, including also Equation (21) rewritten in the notation of the work in [150]:

$$
\begin{gathered}
U_{\mathrm{vdW}}(R)=V_{11}(R)=-\frac{3 E_{I}\left(\alpha_{0}^{(1)}\right)^{2}}{4 R^{6}} ; \\
V_{12}(R)=-\frac{9 E_{I} \alpha_{0}^{(1)} \alpha_{0, \mathrm{E}}^{(2)}}{R^{8}} ; \\
V_{22}(R)=-\frac{504 E_{I}\left(\alpha_{0, \mathrm{E}}^{(2)}\right)^{2}}{40 R^{10}} .
\end{gathered}
$$

Although in the literature all such results are obtained from rigorous calculations within the framework of QED, it is also pointed out, without proof, that "The near-zone result ... may also be obtained with second-order perturbation theory and the electrostatic potentials coupling two electric dipoles ..., an electric dipole and an electric quadrupole ..., and two electric quadrupoles ..." (see in [159], see also in [157], p. 64). Such an approach, which extends London's calculations to higher multipoles as shown by Margenau, does not rely on the quantization of the electromagnetic field.

\section{Unretarded Gravitational van der Waals Forces}

The classical electrostatic dipolar polarizability, $\alpha_{0, \mathrm{E}}^{(1)}$, is typically introduced by means of a classical model in which an electron of mass $m_{e}$, bound to a proton by a spring of constant $K=m_{e} \omega_{0}^{2}$, is displaced by an external electric field, $\mathbf{E}$, so that the position of static equilibrium is given by $-e \mathbf{E}=-K \mathbf{r}$. The corresponding induced dipole moment $\mathbf{p}=e \mathbf{r}$ is, therefore, $\mathbf{p}=e^{2} / m_{e} \omega_{0}^{2} \mathbf{E}$ and the polarizability is, by definition, $\alpha_{0, \mathrm{E}}^{(1)}=e^{2} / m_{e} \omega_{0}^{2}$.

Let us now develop a similar oscillator model to exhibit the static gravitational polarizability, $\alpha_{G}^{(2)}$. It is important to notice at the onset that this calculation differs from the electrostatic case in subtle ways that require careful consideration. For instance, as first pointed out by Szekeres almost half-a-century ago in what was possibly the first attempt to calculate the "gravitational dielectric constant" [160,161], and as later rediscovered by this author in related contexts [162,163], a model of a harmonic oscillator interacting with gravitational waves naturally leads to a Mathieu-type equation of motion. In what follows, as also done by Szekeres, we shall assume that the circumstances possibly triggering parametric resonance are not verified.

We shall again consider an atom in which the electron is connected to the proton by a spring aligned, for instance, along the direction pointing to another identical atom at distance $R$. Again, although the electrodynamical model and this gravitational model appear equivalent, it is not widely appreciated that the evolution of the latter in response 
to external gravitational fields depends critically on the assumed dynamics of the atoms. For instance, the gravitational effects by a mass on "a hydrogen atom whose point proton is immobile" [164] ("Le modèle envisagé est celui d'un atome d'hydrogène dont le proton ponctuel est immobile à l'extérieur de la matière créant le champ" [164]) are drastically different than those on an atom "... in free fall along a geodesic of the spacetime during the time required for an atomic transition" (see in [165], see also in [166]). For the purpose of defining the gravitational polarizability, here we shall assume that both atoms are in free fall although it is clear that, rigorously speaking, this assumption is contradiction with the existence of dispersion forces.

In this 1D model (see in [11], p. 100, Note 11), we shall assume the unperturbed electron to be oscillating harmonically with amplitude equal to $r_{1,0,0}=\frac{3}{2} a_{0}$ so that the position time average is equal to $\bar{z}=r_{1,0,0} / \sqrt{2}$. The displacement from this average due to a gravitational tidal force produced by a mass $M$ is $\overline{\Delta z}=m_{e} R_{0 z 0 z} \bar{z} / K$, where $R_{0 z 0 z}$ are the appropriate components of the Riemann tensor $[167,168]$. This yields the following induced trace-free quadrupole moment tensor $[167,168]$ :

$$
Q_{z z}=\frac{2}{3} m_{e}\left[(\bar{z}+\overline{\Delta z})^{2}-\bar{z}^{2}\right] \simeq \frac{2}{3} m_{e} 2 \bar{z} \overline{\Delta z}=\frac{4}{3} m_{e}^{2} R_{0 z 0 z} \frac{\bar{z}^{2}}{K} .
$$

Therefore, the gravitational polarizability, in the static limit, can now be defined as (Equation (11) in [131]):

$$
\alpha_{0, \mathrm{G}}^{(2)} \equiv \frac{Q_{z z}}{R_{0 z 0 z}}=\frac{4}{3} m_{e}^{2} \frac{\bar{z}^{2}}{K}=\frac{4}{3} m_{e}^{2} \frac{r_{1,0,0}^{2}}{2 K}=\frac{4}{3} m_{e}^{2}\left(\frac{3}{2}\right)^{2} \frac{a_{0}^{2}}{2 K}=\frac{3}{2} \frac{m_{e}^{2} a_{0}^{2}}{K}=\frac{3}{2} \frac{m_{e} a_{0}^{2}}{\omega_{0}^{2}}
$$

where the units also correspond to those given therein (see comment above Equation (32) in [131]).

For the purpose of succinctness, supported by explicit verification with Mathematica, we shall now return to our electrostatic results to extract the expression of the gravitational dispersion force. Obviously, as pointed out by Spruch [57,109], in this case we cannot have a neutral atom. However, the assumption of free fall, by the Principle of Equivalence, implies that all non-tidal gravitational interactions must not affect the dynamics of the system (Notice that introducing a negative mass is a standard procedure employed formally "to eliminate the mass monopole" [169] in analogy with an electric quadrupole. See also Section 6.2). Therefore, the leading contribution left in this unretarded regime is provided by the quadrupole-quadrupole term at Equation (18), upon carrying out Spruch's $\left(e^{2}\right)^{2} \rightarrow$ $\left(G m_{e}^{2}\right)^{2}$ substitution, and with appropriate identification of the gravitational polarizability given above:

$$
V_{22, \text { near }}^{\mathrm{GG}}(R)=-\frac{2835 a_{0}^{8} G^{2} m_{e}^{4}}{4 E_{I} R^{10}}=-\frac{315}{4} \frac{E_{I} G^{2}\left(\alpha_{0, \mathrm{G}}^{(2)}\right)^{2}}{R^{10}} .
$$

This equation, obtained by quantizing the interacting oscillators but with classical gravitation, is identical to that found by Ford, Hertzberg, and Karouby (see Equation (31) in [131]) from quantum field theory provided that the two atoms are identical and with $E_{I}=\hbar \omega_{0}$.

Finally, as also hinted to by Spruch, we provide new results, that is, the expressions for the mixed quadrupole-quadrupole and dipole-quadrupole electric-gravitational potentials in the unretarded regime. These can be obtained analogously to what done above by carrying out only one $e^{2} \rightarrow G m_{e}^{2}$ substitution while leaving one $e^{2}$ factor unchanged in Equations (18) and (19), respectively:

$$
V_{22, \text { near }}^{\mathrm{EG}}(R)=-\frac{2835 a_{0}^{8} e^{2} G m_{e}^{2}}{2 E_{I} R^{10}}=-\frac{63 E_{I} G \alpha_{0, \mathrm{E}}^{(2)} \alpha_{0, \mathrm{G}}^{(2)}}{R^{10}} .
$$




$$
V_{12, \text { near }}^{\mathrm{EG}}(R)=-\frac{135 a_{0}^{6} e^{2} G m_{e}^{2}}{E_{I} R^{8}}=-\frac{45 E_{I} G \alpha_{0, \mathrm{E}}^{(1)} \alpha_{0, \mathrm{G}}^{(2)}}{2 R^{8}} .
$$

Notice, however, that the explicit Mathematica calculation shows a factor of 2 difference in addition to the $e^{2} \rightarrow G m_{e}^{2}$ substitution.

\section{McLachlan's Semiclassical Calculation of Electrodynamical Dispersion Forces}

As we have mentioned above (Section 1), McLachlan developed an approach to the calculation of dispersion forces between polarizable bodies requiring only the quantization of the atomic degrees of freedom while leaving the interacting electromagnetic field classical $[49,170]$. Within this semiclassical framework, all results from the Lifshitz theory, including the potential of two or more molecules or that of infinite parallel dielectric surfaces, both in the unretarded and retarded regimes, are correctly recovered. As typical, a method that "... gives the main results of Lifshitz's treatment and does not use quantum field theory" is justified merely as having "the advantage of using simple physical concepts" [171]. In the case of this paper, however, we intend to employ this strategy to show that a completely classical gravitational field is expected to produce dispersion forces equal to those predicted in the low-energy limit of a hypothetical theory of quantum gravity. Therefore, a hypothetical experimental verification of the existence of such forces would not represent proof that the gravitational field is quantized. For the purpose of considering such a generalization of McLachlan's theory to the gravitational case, we first focus on the electromagnetic case, sketching his calculation of the interatomic dipole-dipole dispersion potential, which leads back to the expressions by London and by Casimir and Polder. We then broaden this treatment to dipole-quadrupole and quadrupole-quadrupole electrodynamical dispersion forces in simplified 1D models to highlight the physical principles; thus, reaching expressions obtained approximately four decades after Casimir and Polder by Thirunamachandran [172] from non-relativistic QED [79], later in collaboration with Salam, Power, and Jenkins [150,156,157,159,173].

\subsection{Electric Dipole-Electric Dipole Dispersion Forces}

Here, we closely follow the analyses of the relevant physical arguments in McLachlan's approach provided by Margenau (see in [50], especially Section 6.3), by Renne [174,175], and by Langbein [42], adapting all notation and definitions therein to compare our results to those above and elsewhere in the published literature, and to extend this treatment to the case of gravitational dispersion forces.

Let us consider two coupled harmonic oscillators of displacement components $u_{A, i}$ and $u_{B, j}$ of masses $m_{A, B}$ and natural frequencies $\omega_{A, B}$. By writing the equation of motion of each oscillator in the absence of friction and under the action of an external force $F_{\text {ext }}=$ $F(\omega) \exp (-i \omega t)$, with $i=\sqrt{-1}$, the usual amplitude of the forced oscillation is found, $u_{A, B}=\chi_{A, B}(\omega) F_{\text {ext }}$, where $\chi_{A, B}(\omega)$ is the generalized scalar susceptibility (As discussed below, the dimensionality of this definition differs from that at Equation (10) by [Charge $]^{2}$. For this reason, we add a factor $e^{2}$ to the force equation, $F_{B A, j}=u_{A, i} e^{2} T_{i j}(\omega)$, as explicitly done in all treatments following that by London [17] (for instance, see Equations (3.67-68) in [11]). The definitions in McLachlan are consistent with those by Langbein (compare Equation (5.3) in [170] with our Equation (10)). On the other hand, Margenau adopts our definition at Equation (10) (see Ch. 2, Equation (83) in [50])):

$$
\chi_{j}(\omega)=\frac{1}{m_{j}\left(\omega_{j}^{2}-\omega^{2}\right)} .
$$

In the hypothesis of linear oscillator coupling, the force $F_{B A, j}$ exerted by oscillator $A$ on $B$ is given by the displacement $u_{A, i}$ as $F_{B A, j}=u_{A, i} e^{2} T_{i j}(\omega)$, where $T_{i j}(\omega)$ will represent the classical interaction tensor and $T_{i j}(\omega)=T_{j i}(\omega)$ due to Newton's third law. By writing the equations of motion for the two oscillators and assuming solutions of the form $u_{A, B}=$ $u_{A, B}(\omega) \exp (-i \omega t)$, with $i=\sqrt{-1}$, we find the standard homogeneous system of two 
equations whose determinant, set equal to zero, yields the secular equation for the normal modes [176]:

$$
\left(\omega^{2}-\omega_{A}^{2}\right)\left(\omega^{2}-\omega_{B}^{2}\right)=e^{4} \frac{1}{m_{A}} T_{i j} \frac{1}{m_{B}} T_{j i} .
$$

At this point, as clearly articulated by London (see in [17], § 4; see also in [22,46,47,177]), the quantization of the oscillation modes of the harmonic oscillator, consistently with the Uncertainty Principle, leads to a non-vanishing ground state energy, which is shifted by the interatomic coupling according to the following expression,

$$
\Delta E=\frac{1}{2} \hbar\left(\Omega_{A}+\Omega_{B}\right)-\frac{1}{2} \hbar\left(\omega_{A}+\omega_{B}\right),
$$

where $\Omega_{A, B}$ are the solutions of the secular equation. By solving Equation (30) and expanding the result in the parameter $T_{i j} T_{j i} / m_{A} m_{B}$, we find

$$
\Delta E=-\frac{e^{4}}{4} \hbar \frac{1}{m_{A}} T_{i j} \frac{1}{m_{B}} T_{j i} \frac{1}{\omega_{A} \omega_{B}\left(\omega_{A}+\omega_{B}\right)} .
$$

In order to verify this result, let us first discuss the structure of the dipole interaction tensor $\mathbf{T}_{\mathbf{i j}}$ in the unretarded case. In the present system, the electrostatic potential at $\mathbf{r}_{\mathbf{B}}$ of a dipole $A$ located at $\mathbf{r}_{\mathbf{A}}$ is given by the elementary expression $[178,179]$ :

$$
U_{\text {dip }}=-e \mathbf{u}_{A} \cdot \nabla_{A}\left(\frac{1}{\left|\mathbf{r}_{\mathbf{A}}-\mathbf{r}_{\mathbf{B}}\right|}\right)
$$

so that the force acting on the elastically bound electron of dipole $B$ is

$$
F_{B A, j}=-e^{2} u_{A, i} \nabla_{A, i} \nabla_{B, j}\left(\frac{1}{\left|\mathbf{r}_{\mathbf{A}}-\mathbf{r}_{\mathbf{B}}\right|}\right)
$$

and the the dipole interaction tensor can be read out as

$$
\mathbf{T}_{\mathbf{i j}}=-\nabla_{A, i} \nabla_{B, j} \frac{1}{\left|\mathbf{r}_{\mathbf{A}}-\mathbf{r}_{\mathbf{B}}\right|} .
$$

A generalization of the quantity $T_{i j} T_{j i}$ to the full 3D case requires the explicit calculation of the quantity $\operatorname{tr}\left(\mathbf{T}_{\mathbf{i j}} \mathbf{T}_{\mathbf{j i}}\right)=6 / R_{A B}^{6}$, where $R_{A B}=\left|\mathbf{r}_{\mathbf{A}}-\mathbf{r}_{\mathbf{B}}\right|$. Therefore, for $\omega_{A}=$ $\omega_{B}=\omega_{0}$ and $m_{A}=m_{B}=m_{e}$, we finally have

$$
\Delta E(R)=U_{\mathrm{vdW}}(R)=-\frac{3 \hbar e^{4}}{4 m_{e}^{2} \omega_{0}^{3} R_{A B}^{6}}=-\frac{3 \hbar^{4} e^{4}}{4 m_{e}^{2} E_{I}^{2}} \frac{1}{E_{I} R_{A B}^{6}}=-\frac{3 a_{0}^{4} e^{4}}{E_{I} R_{A B}^{6}},
$$

as already found at Equation (6) and where, again, $E_{I}=\hbar \omega_{0}=e^{2} / 2 a_{0}$ and $a_{0}=\hbar^{2} / m_{e} e^{2}$.

Importantly, a different route is to express the natural frequencies at Equation (32) in integral form by means of the identity:

$$
\frac{1}{\omega_{A} \omega_{B}\left(\omega_{A}+\omega_{B}\right)}=\frac{1}{\pi} \int_{-\infty}^{\infty} d \omega \frac{1}{\left(\omega^{2}+\omega_{A}^{2}\right)\left(\omega^{2}+\omega_{B}^{2}\right)},
$$

so that, equivalently, by integrating along the imaginary frequency axis, where $\omega_{C}=$ $\omega_{R}+i \omega_{I}$ :

$$
\left.\left.\Delta E=-\frac{\hbar}{2 \pi} \int_{0}^{\infty} d \omega_{I} e^{2} \chi_{A}\left(i \omega_{I}\right) T_{i j} e^{2} \chi_{B}\left(i \omega_{I}\right) T_{j i}=-\frac{\hbar}{2 \pi} \int_{0}^{\infty} d \omega_{I} \alpha_{A, \mathrm{E}}^{(1)}\right)\left(i \omega_{I}\right) T_{i j} \alpha_{B, \mathrm{E}}^{(1)}\right)\left(i \omega_{I}\right) T_{j i} .
$$

Again, a generalization of this result to fully independent three-dimensional (3D) oscillators leads to the well-known result by McLachlan in terms of polarizability tensors:

$$
\left.\left.\Delta E=-\frac{\hbar}{2 \pi} \int_{0}^{\infty} d \omega_{I} \operatorname{tr}\left(\alpha_{A, \mathrm{E}}^{(1)}\right)\left(i \omega_{I}\right) \cdot \mathbf{T}_{i j} \cdot \alpha_{B, \mathrm{E}}^{(1)}\right)\left(i \omega_{I}\right) \cdot \mathbf{T}_{j i}\right) .
$$


In the case of isotropic molecules, the polarizability tensors again reduce to scalars and we can write, in the unretarded case:

$$
\left.\left.\Delta E=-\frac{3 \hbar}{\pi} \frac{1}{R_{i j}^{6}} \int_{0}^{\infty} d \omega_{I} \alpha_{A, \mathrm{E}}^{(1)}\right)\left(i \omega_{I}\right) \alpha_{B, \mathrm{E}}^{(1)}\right)\left(i \omega_{I}\right) .
$$

In order to consider the retarded case, we must again compute the force on dipole $B$ by means of the dipole classical radiation fields. This can be done starting from the dipole Hertz vector, $\mathbf{Z}\left(\mathbf{r}_{\mathbf{A}}, \mathbf{r}_{\mathbf{B}}, \omega\right)$, given by (see in [178], Sections 14.5-7):

$$
\mathbf{Z}\left(\mathbf{r}_{\mathbf{A}}, \mathbf{r}_{\mathbf{B}}, \omega\right)=\mathbf{p}_{\mathbf{A}} \frac{e^{i(\omega / c)\left|\mathbf{r}_{\mathbf{A}}-\mathbf{r}_{\mathbf{B}}\right|}}{\left|\mathbf{r}_{\mathbf{A}}-\mathbf{r}_{\mathbf{B}}\right|}
$$

The electric field is then found by means of the auxiliary vector, $\mathbf{C}=\nabla \times \mathbf{Z}$, as $\mathbf{E}=$ $\nabla \times \nabla \times \mathbf{Z}$. In Cartesian coordinates, this leads to the following expression for the tensor to be multiplied by $\mathbf{p}_{\mathbf{A}}=e u_{A, i}$ in order to obtain the force on dipole $B, F_{B A, j}=u_{A, i} e^{2} T_{i j}(\omega)$,

$$
\mathbf{T}_{\mathbf{i j}}=\left[\nabla_{i} \nabla_{j}-\delta_{i j} \nabla^{2}\right] \frac{e^{i(\omega / c)\left|\mathbf{r}_{\mathbf{A}}-\mathbf{r}_{\mathbf{B}}\right|}}{\left|\mathbf{r}_{\mathbf{A}}-\mathbf{r}_{\mathbf{B}}\right|},
$$

where all derivatives are respect to the $\mathbf{r}_{\mathbf{A}}=\left(x_{A}, y_{A}, z_{A}\right)$ coordinates. Let us now assume, without loss of generality, dipole $A$ to be at the origin $\left(\mathbf{r}_{\mathbf{A}}=\mathbf{0}\right)$ and dipole $B$ at $\mathbf{r}_{\mathbf{B}}=$ $(r, 0,0)$. Direct calculation in this geometry shows that the only non-zero components of the interaction tensor are, after the customary rotation to the imaginary frequency axis (see Equation (101) in [50]):

$$
\begin{aligned}
& \mathbf{T}_{\mathbf{1 1}}=\left(\frac{2}{R^{3}}\right)\left(1+R \frac{\omega_{I}}{c}\right) e^{-R \omega_{I} / c} \\
& \mathbf{T}_{\mathbf{2 2}}=\mathbf{T}_{\mathbf{3 3}}=-\left(\frac{1}{R^{3}}\right)\left(1+R \frac{\omega_{I}}{c}+R \frac{\omega_{I}^{2}}{c^{2}}\right) e^{-R \omega_{I} / c} .
\end{aligned}
$$

Therefore, for isotropic atoms, the dipole polarizabilities are again scalars and the trace at Equation (39) becomes

$$
\operatorname{tr}\left(\mathbf{T}_{\mathbf{i j}} \mathbf{T}_{\mathbf{j i}}\right)=e^{-2 R \omega_{I} / c}\left(\frac{2 \omega_{I}^{4}}{c^{4} R^{2}}\right)\left(1+\frac{2 c}{\omega_{I} R}+\frac{5 c^{2}}{\omega_{I}^{2} R^{2}}+\frac{6 c^{3}}{\omega_{I}^{3} R^{3}}+\frac{3 c^{4}}{\omega_{I}^{4} R^{4}}\right)
$$

By using this result and Equation (10) in Equation (39), we find

$$
\begin{aligned}
\Delta E(R)=-\frac{\hbar}{2 \pi}\left(\frac{2}{3 \hbar}\right)^{2}\left(\frac{2}{c^{4} R^{2}}\right) \sum_{r, s} \omega_{r, 0} \omega_{s, 0}\left\|\mu_{0, r}\right\|^{2}\left\|\mu_{0, s}\right\|^{2} & \int_{0}^{\infty} d \omega_{I} \frac{\omega_{I}^{4} e^{-2 R \omega_{I} / c}}{\left(\omega_{r, 0}^{2}+\omega_{I}^{2}\right)\left(\omega_{s, 0}^{2}+\omega_{I}^{2}\right)} \times \\
& \left(1+\frac{2 c}{\omega_{I} R}+\frac{5 c^{2}}{\omega_{I}^{2} R^{2}}+\frac{6 c^{3}}{\omega_{I}^{3} R^{3}}+\frac{3 c^{4}}{\omega_{I}^{4} R^{4}}\right) .
\end{aligned}
$$

This expression for the dispersion force in terms of the dynamical polarizability as a function of the imaginary frequency was first obtained by Casimir and Polder [24] (see also Equation (42) in [50] and Equation (3.87) in [11,180]).

In the simplified case of two identical atoms in which only one transition is dominant and in the unretarded limit $\left(e^{-2 R \omega_{I} / c} \rightarrow 1\right)$, only the integral corresponding to the last term $\propto R^{-4}$ in Equation (45) must be considered so that we recover the well-known expression at Equation (21):

$$
\Delta E(R)=U_{\mathrm{vdW}}(R)=-\frac{3 \hbar}{\pi}\left(\frac{2}{3 \hbar}\right)^{2}\left(\frac{1}{R^{6}}\right) \omega_{0}^{2}\left\|\mu_{0, r}\right\|^{4}, \int_{0}^{\infty} d \omega_{I} \frac{1}{\left(\omega_{0}^{2}+\omega_{I}^{2}\right)^{2}}=-\frac{3 \hbar \omega_{0}\left(\alpha_{0, \mathrm{E}}^{(1)}\right)^{2}}{4 R^{6}},
$$


where we used the expression for the static polarizability in terms of the dipole matrix element equivalent to that given above immediately preceding Equation (20), $\alpha_{0, \mathrm{E}}^{(1)}=\frac{2}{3}\left\|\mu_{0, r}\right\|^{2} / \hbar \omega_{0}$.

The famous limit of the result at Equation (46) referred to as the Casimir-Polder potential can be obtained by realizing that, in the fully retarded regime, the static polarizability provides the leading contribution (See footnote 14, p. 104 of the work in [11]) so that, by using Equation (45), Equation (39) becomes

$$
\Delta E(R)=U_{\mathrm{CP}}(R)=-\frac{\hbar}{2 \pi}\left(\alpha_{0, \mathrm{E}}^{(1)}\right)^{2} \int_{0}^{\infty} d \omega_{I} \operatorname{tr}\left(\boldsymbol{T}_{i j} \cdot \boldsymbol{T}_{j i}\right)=-\frac{23 \hbar c}{4 \pi R^{7}}\left(\alpha_{0, \mathrm{E}}^{(1)}\right)^{2} .
$$

Importantly for our further generalizations, in the isotropic case, Equation (46) can be rewritten by means of Equation (42) as [156,181]:

$$
\left.\left.\Delta E=-\frac{\hbar}{2 \pi} \int_{0}^{\infty} \alpha_{A, \mathrm{E}}^{(1)}\right)\left(i \omega_{I}\right) \alpha_{B, \mathrm{E}}^{(1)}\right)\left(i \omega_{I}\right)\left[\left(\nabla_{i} \nabla_{j}-\delta_{i j} \nabla^{2}\right) \frac{e^{-R \omega_{I} / c}}{R}\right]\left[\left(\nabla_{i} \nabla_{j}-\delta_{i j} \nabla^{2}\right) \frac{e^{-R \omega_{I} / c}}{R}\right] d \omega_{I} .
$$

\subsection{Application to Dispersion Forces with Electric Quadrupoles: One-Dimensional Case}

As anticipated by McLachlan, the above strategy can be generalized to the case of atoms interacting through higher order electric and magnetic multipole fields (see in [49], Section 7 and Appendix). To the best of this author's knowledge, however, such a semiclassical program has never been appeared in the published literature. In what follows, in support of our analysis of the gravitational case, we consider electric dipole-electric quadrupole and electric quadrupole-electric quadrupole interactions. In order to expose the physical processes involved behind this approach while avoiding lengthy calculations, we shall not analyze the full 3D geometry but, as often done in survey presentations about the unretarded and retarded regimes $[46,47,177]$, we shall restrict ourselves to the 1D case. Extension to the full 3D case is promptly achieved through straightforward, though technically more intricate, calculations as just demonstrated in the previous section.

In analogy with the process leading to Equation (36), let us now consider one simple 1D classical quadrupole, $A$, represented by two pairs of identical, opposing, elastically bound point dipoles, $\mathbf{p}_{A} / \delta$ at a mutual vector distance $\delta \mathbf{u}_{\mathbf{A}}$, where $\delta$ is a limit parameter (Section 4.4 in [182]) (Another useful simple quadrupole model is given by three charges $(+q,-2 q,+q)$ arranged along a straight line at charge-to-charge distances equal to $d$ [169]). Let us first assume the two opposing dipoles to be aligned along the $x$-axis and interacting with another single dipole, $\mathbf{p}_{B}$, like those used in the previous section, also parallel to the the $x$-axis.

The only non-vanishing component of the point quadrupole tensor, in the limit $\delta \rightarrow 0$, is $\mathcal{Q}_{x x}=u_{A} p_{A}$ and the traceless quadrupole tensor becomes $Q_{x x}=3 \mathcal{Q}_{x x}-\mathcal{Q}_{x x}=2 \mathcal{Q}_{x x}=$ $2 u_{A} p_{A}$. The quadrupole potential can then be written as [183]:

$$
U_{\text {quad }}=\frac{1}{6} Q_{x x} \frac{\partial^{2}}{\partial x^{2}}\left(\frac{1}{\left|\mathbf{r}_{\mathbf{A}}-\mathbf{r}_{\mathbf{B}}\right|}\right)
$$

and, analogously to Equation (34), the force on the oscillating charge of dipole $\mathbf{p}_{B}$ is:

$$
F_{B A, x}=\frac{1}{3} e u_{A} p_{A} \frac{\partial^{3}}{\partial x^{3}}\left(\frac{1}{\left|\mathbf{r}_{\mathbf{A}}-\mathbf{r}_{\mathbf{B}}\right|}\right) .
$$

Finally, since the $1 \mathrm{D}$ 'trace' $T_{i j} T_{j i}=\left(\partial^{3} / \partial x_{A}^{3}\right)\left(1 /\left|x_{A}-x_{B}\right|\right)=36 / x^{8}$, we obtain, indicating $r=\left|x_{A}-x_{B}\right|$ and by identifying $p_{A}^{2}=\frac{1}{3}|| \mu_{1,0,0}||^{2}=a_{0}^{2} e^{2}$.

$$
\Delta E_{12}^{1 \mathrm{D}}=-\frac{e^{4}}{4} \hbar \frac{1}{9} e^{2}\left\|p_{A}\right\|^{2} \frac{1}{2 \omega_{0}^{3}} \frac{1}{m_{e}^{2}} \frac{36}{x^{8}}=-6 \frac{a_{0}^{6} e^{4}}{E_{I}} \frac{1}{r^{8}},
$$


to be compared to Equation (14). By again exploiting the identity at Equation (37) and by comparing the middle term of the above equation to that at Equation (36), it can be recognized that $\left.\left\|\mu_{1,0,0}\right\|^{2} \chi_{A}\left(i \omega_{I}\right) T_{i j}=\alpha_{B, \mathrm{E}}^{(2)}\right)$ and $\left.e^{2} \chi_{B}\left(i \omega_{I}\right) T_{j i}=\alpha_{B, \mathrm{E}}^{(1)}\right)$ (see Equations (1.7)-(1.8) in [150]), so that this result can be put into the important integral form analogous to that as at Equation (38)

$$
\left.\left.\left.\left.\Delta E_{12}=V_{12}(r)=-\frac{\hbar}{2 \pi} \int_{0}^{\infty} d \omega_{I} \alpha_{A, \mathrm{E}}^{(1)}\right)\left(i \omega_{I}\right) T_{i j} \alpha_{B, \mathrm{E}}^{(2)}\right)\left(i \omega_{I}\right) T_{j i}=-\frac{18 \hbar}{\pi} \frac{1}{r^{8}} \int_{0}^{\infty} d \omega_{I} \alpha_{A, \mathrm{E}}^{(1)}\right)\left(i \omega_{I}\right) \alpha_{B, \mathrm{E}}^{(2)}\right)\left(i \omega_{I}\right)
$$

As mentioned above (Section 4.2), this result was previously obtained by a full QED approach by Jenkins (Equation (6.7), Ref. [159]), and then re-derived by appropriate generalizations by Salam (Equation (3.5) in [156]) and by Power (Equation (1.5) in [150]) all working with Thirunamachandran (Notice that units employed in these works differ from one another. Power and Thirunamachandran showed that the correct numerical factor in the full 3D case is 90. Also, the speed of light $c$ factors in Equations (1.4)-(1.6) in [150], which refer to the unretarded limit, are erroneous).

In the case of electric unretarded electric quadrupole-quadrupole forces, the system is represented by two quadrupoles as $A$ above, that is, a total of four dipoles elastically bound in opposing pairs and, in the 1D case, all parallel to the $x$-axis. The force due to the electric field produced by quadrupole $A$ acting on the oscillating dipole of atom $B$ is given by the well-known equation $\mathbf{F}=(\mathbf{p} \cdot \nabla) \mathbf{E}[179,182]$ so that the force becomes

$$
F_{B A, x}=-\frac{1}{3} e u_{A} p_{A} p_{B} \frac{\partial^{4}}{\partial x^{4}}\left(\frac{1}{\left|\mathbf{r}_{\mathbf{A}}-\mathbf{r}_{\mathbf{B}}\right|}\right)
$$

Therefore, $T_{i j} T_{j i}=\left(\partial^{4} / \partial x_{A}^{4}\right)\left(1 /\left|x_{A}-x_{B}\right|\right)=576 / x^{10}$, leading to the unretarded potential

$$
\Delta E_{22}^{1 \mathrm{D}}=-32 \frac{a_{0}^{8} e^{4}}{E_{I}} \frac{1}{r^{10}}
$$

again to be compared to Equation (14). The integral form immediately descends from this formulation as

$$
\left.\left.\Delta E_{22}=V_{22, \text { near }}(r)=-\frac{288 \hbar}{\pi} \frac{1}{r^{10}} \int_{0}^{\infty} d \omega_{I} \alpha_{A, \mathrm{E}}^{(2)}\right)\left(i \omega_{I}\right) \alpha_{B, \mathrm{E}}^{(2)}\right)\left(i \omega_{I}\right),
$$

again in agreement, to within the constant numerical factor, with results previously found $[150,156,159]$. Importantly, a comparison of the quantity $T_{i j} T_{j i}$ at Equation (35) and in the cases above shows that the required order of the derivative of the potential $1 /\left|\mathbf{r}_{\mathbf{A}}-\mathbf{r}_{\mathbf{B}}\right|$ increases by one unit for each integer order of the multipoles considered. This observation is reflected in the generalization of the results above to all multipole orders, including possibly anisotropic polarizabilities. For instance, to treat the general case of any interatomic distance, the factors $\left[\left(\nabla_{i} \nabla_{j}-\delta_{i j} \nabla^{2}\right)\left(e^{-R \omega_{I} / c} / R\right)\right]$ at Equation (49) must likewise be modified into $\left[\left(\nabla_{i} \nabla_{j}-\delta_{i j} \nabla^{2}\right) \nabla_{k}\left(e^{-R \omega_{I} / c} / R\right)\right]$ and $\left[\left(\nabla_{i} \nabla_{j}-\delta_{i j} \nabla^{2}\right) \nabla_{k} \nabla_{l}\left(e^{-R \omega_{I} / c} / R\right)\right]$ in the two systems analyzed, respectively, and "tumbling averages" must be taken over all orientations in the case of isotropic atoms (see Equation (2.9) in [156]).

To briefly illustrate the important fully retarded regime, let us consider the interactions $\Delta E_{12}^{1 \mathrm{D}}$ (Equation (52) and $\Delta E_{22}^{1 \mathrm{D}}$ (Equation (55) by starting from the quadrupole field Hertz vector $j$-component (see in [178], Section 14-8), as done above at Equation (41):

$$
Z_{\text {quad }, j}=\frac{\omega}{2 c} \frac{e^{i(\omega / c)\left|\mathbf{r}_{\mathbf{A}}-\mathbf{r}_{\mathbf{B}}\right|}}{\left|\mathbf{r}_{\mathbf{A}}-\mathbf{r}_{\mathbf{B}}\right|^{2}} x_{i} \mathcal{Q}_{i j} .
$$

By proceeding again as above to calculate the electric field as $\mathbf{E}=\nabla \times \nabla \times \mathbf{Z}_{\text {quad }}$, analogously to Equation (45), by forming the trace of the interaction tensor in this 1D 
model, isolating the static polarizabilities $\left.\alpha_{0, \mathrm{E}}^{(1)}\right)$ and $\left.\alpha_{0, \mathrm{E}}^{(2)}\right)$, and by calculating an integral analogous to that at Equation (48), we find, for the dipole-quadrupole energy,

$$
\left.\left.\Delta E_{12}=V_{12, \text { far }}(r)=-C_{12} \frac{\hbar c}{\pi r^{9}} \alpha_{0, \mathrm{E}}^{(1)}\right) \alpha_{0, \mathrm{E}}^{(2)}\right)
$$

where $C$ is a numerical constant. A similar procedure for the quadrupole-quadrupole interaction, seeking the quantity $(\mathbf{p} \cdot \nabla) \mathbf{E}$, leads to:

$$
\left.\left.\Delta E_{22}=V_{22, \text { far }}(r)=-C_{22} \frac{\hbar c}{\pi r^{11}} \alpha_{0, \mathrm{E}}^{(2)}\right) \alpha_{0, \mathrm{E}}^{(2)}\right) .
$$

These results are in full agreement, to within the constants $C_{12}$ and $C_{22}$, with those by Power and Thirumanamachandran (Equations (4.13)-(4.14) in [150]). Furthermore, the corresponding expressions in terms of integrals over the proper multipole polarizabilities as functions of the complex frequency, generalizing Equation (49), are promptly written and lead to the same expressions provided by Salam and collaborators (a full review is provided in [157] and references therein).

\subsection{Application of McLachlan's Approach to Gravitational Dispersion Forces}

The semiclassical computation of gravitational dispersion forces is a straightforward extension of the process just described. As we have already commented (Sections 5 and 6.2), strong analogies exist between the calculations with multipoles in the electrodynamic and in the gravitational case [169]. The calculation of the multipole fields in Cartesian coordinates in both electromagnetism and gravitation is mathematically relatively burdensome, as shown even in the much simplified 1D case, and it benefits from the computer algebra approach applied extensively in this paper. However, the physical principle of McLachlan's approach as applied to gravitation within the context of general relativity is quite clear. In analogy to electrodynamics, atoms interact through possibly retarded gravitational fields due to the oscillating sources and dispersion forces appear as the atomic energy levels are quantized, that is, one again envisions two systems of elastically bound dipole pairs aligned to the $x$-axis in near-free fall (considering accelerations due to dispersion forces as much smaller than that due to the monopoles). As shown in Section 5 in our simplified 1D model, the force acting on each oscillator is given by the appropriate Riemann tensor component, which can be obtained from the classical field metric solution given in general, for instance, by Ford et al. (Equations (4)-(9) in [131]) or, for a simplified model, by Price et al. (Equations (24)-(26) in [169]). Importantly, in the gravitational case, the Riemann tensor, and thus the interaction tensor, are determined through second derivatives with respect to spacetime coordinates of the metric tensor solution (Equations (7.27)-(7.29) in [184]), which does not directly determine the force. As in the electrodynamical case, this computation reproduces our results for the unretarded dispersion force in Section 5 and Ford's results in all distance regimes, to within numerical constants reflecting our dimensional simplification.

\section{Experimental Detection of Gravitational Dispersion Forces}

There is no doubt that identifying any effects of gravitation on dispersion forces is one of the most extreme contemporary experimental challenges. Generally speaking, these include not only the gravitational dispersion forces discussed in this paper but also the effects of time-independent spacetime curvature on electrodynamical dispersion forces, which can be viewed as a manifestation of the inertial equivalent of the Casimir potential energy [88,185]. Although these two avenues for gravitation to affect theoretical predictions are sometimes, puzzlingly, conflated [186], they are connected to drastically different phenomena. Whereas treatments of the latter hinge on relatively well-understood, uncontroversial concepts and measurements are deemed within reach [187-189], the former, which we briefly consider herein, depend on novel, exotic physical mechanisms. 
As mentioned above (Section 3), the possibility to detect a macroscopic gravitational Casimir force has been recently brought to the fore [129] by the proposal that superconductors could be caused to act as nearly-ideal gravitational wave reflectors through a so-called "Heisenberg-Coulomb effect" [125]. This is a remarkably bold statement considering that even neutron stars are predicted to reflect gravitational waves with an index of reflection of only $4-25 \%$ [190]. The idea that superconductors might appreciably reflect and refract gravitational waves, thus enabling table-top gravitational wave optics [122,124], has been proposed by several authors [191] but it remains experimentally unverified. As already clarified by this author, in the theory of the Heisenberg-Coulomb effect, all radiation fields are treated classically [125] and the gravitational Casimir effect, even if detected, would not represent proof of the quantization of the gravitational field as extensively discussed in this paper [60]. Furthermore, initial predictions of the gravitational Casimir force as being even larger than its QED counterpart by almost one order of magnitude were shown to be due to a computational error [130]. Finally, recent measurements of the Casimir force between superconductors [186], although criticized [192], were reported to rule out the predicted gravitational Casimir effect in superconductors.

As regards systems in which detection of a gravitational Casimir-Polder effect might be feasible, Ford and collaborators [131] speculatively suggest "microscopic clumps built out of heavy sterile neutrinos" as possible, but as yet unconfirmed, interacting dark matter systems. An additional suggestion has been made by the present author to study Efimov states in dark matter once and if its components are definitely identified [193].

A more optimistic consideration is that identifying experimentally accessible phenomena that involve mixed potentials would represent an immense step towards fulfilling a detection goal since such quantities are larger by approximately 43 orders of magnitude $\left(\sim e^{2} / G m_{e}^{2}\right)$ than those due purely to gravitational fluctuations. This exploration is only now starting with indications, for instance, that low energy scattering experiments with neutrons in ground-based laboratories may prove useful [194].

As we have argued throughout this paper, any determination of the existence of gravitational dispersion forces will probably not represent conclusive proof of the quantum nature of the gravitational field. However, this makes the prospect of such an experiment in no way less exciting. What shall we find? Surprising scenarios are indeed possible, especially in the even more challenging retarded regime in which the existence of a minimal length may introduce modifications in the expressions found herein. Any theoretical estimates might be modified if fundamental assumptions are found to be inaccurate, for instance, because of a significant stochastic gravitational wave background [60] or the existence of Planck scale granularity [194]. Regardless of whether gravitational dispersion force behavior departs from or conforms to theoretical predictions, we shall enter a new era in our understanding of crucial parameters that characterize the structure of spacetime as well as the universe on astrophysical and cosmological scales.

\section{Discussion and Conclusions}

In closing, it is appropriate to discuss the connection between the main thesis of this paper and two apparently widely separate issues. Philosophically speaking, the history of forces between the basic constituents of matter is far older than that of the last few decades. In fact, interactions between "atoms" were deemed indispensable to the apparatus of philosophical atomism introduced by Leucippus and Democritus of Abdera. As this author has previously discussed [195], the existence of dispersion forces, whether within a quantum or a semiclassical description, ties the logical self-consistency of atomistic philosophy to the question of the nature of the vacuum. The challenge, as identified by Post, appears to be “... the difficulty of reconciling a world of interacting parts with atomism, which ideally requires independence for its atoms" [196]. As we have seen, physics, even at a semiclassical level, explains the existence of dispersion forces by introducing the concept of zero-point field, thus making a statement about another difficulty "...traditionally associated with atomism, ... the problem of the void". J. A. Wheeler stated: "No point is more central than 
this, that empty space is not empty..." [167] and adopting his "foam-like structure" [197] must necessarily introduce new interactions among the basic constituents of matter. Atomism, identified by some with determinism [198], functions if we accept stochasticity or uncertainty so as to introduce needed forces between polarizable particles. Therefore, the subject of gravitational dispersion forces, by introducing uncertainty in spacetime, adds one further layer of complexity to these reflections, as will be discussed elsewhere.

Technologically speaking, it would be natural to doubt that such fantastically small interactions as gravitational dispersion forces might lead to novel applications. Although we shall not even attempt to speculate about possible future developments here, we shall recall that, when Jordan Maclay and his collaborators suggested in 1995 that electrodynamical Casimir forces may have some important applications in microelectromechanical system (MEMS) engineering [70], it was certainly less than obvious that such apparently exotic interactions would, two decades later, enable a human to climb vertically on glass [199]. Such has been, however, the history of dispersion force research technology transfer [3].

Funding: This research received no external funding.

Institutional Review Board Statement: "Not applicable" for studies not involving humans or animals. Informed Consent Statement: "Not applicable" for studies not involving humans.

Acknowledgments: An early sketch of the ideas fully developed in this paper was presented as an oral paper at the 4th Symposium on the Casimir Effect held in St. Petersburg [200]. It is my pleasure to acknowledge enlightening email correspondence with Professor Akbar Salam (Wake Forest University) on his work on long-range intermolecular forces. I am grateful to the issue editor, Professor Maclay, for his gracious invitation to contribute this article.

Conflicts of Interest: The authors declare no conflicts of interest.

\section{References}

1. Simpson, W.M. Ontological aspects of the Casimir Effect. Stud. Hist. Philos. Mod. Phys. 2014, 48, 84-88. [CrossRef]

2. Casimir, H.B.G. On the attraction between two perfectly conducting plates. Proc. Kon. Ned. Akad. Wetenshap 1948, 51, 793-795.

3. Pinto, F. The future of van der Waals force enabled technology-transfer into the aerospace marketplace. In Nanotube Superfiber Materials, Science to Commercialization; Schulz, M.J., Shanov, V.N., Yin, J., Cahay, M., Eds.; Elsevier: Amsterdam, The Netherlands, 2019; Chapter 29, pp. 729-794.

4. Sakurai, J.J. Advanced Quantum Mechanics; Addison-Wesley Publ. Co.: Redwood City, CA, USA, 1987.

5. Planck, M. Über die Begründung des Gesetzes der schwarzen Strahlung. Ann. Phys. 1912, 342, 642-656. [CrossRef]

6. Einstein, A.; Stern, O. Einige Argumente für die Annahme einer molekularen Agitation beim absoluten Nullpunkt. Ann. Phys. 1913, 345, 551-560. [CrossRef]

7. Bohr, N. Niels Bohr Collected Works; North-Holland Publishing Company: Amsterdam, The Netherlands, 1981 ; Volume 2.

8. Nernst, W. Uber einen versuch, von quantentheoretischen Betrachtungen zur Annahme stetiger Energieanderungen zuruckzukehren. Verhandlungen Dtsch. Phys. Ges. 1916, 18, 83-117.

9. Chu, B. Molecular Forces, Based on the Baker Lectures of Peter J. W. Debye; John Wiley \& Sons: New York, NY, USA, 1967.

10. Kuhn, T.S. Black-Body Theory and the Quantum Discontinuity, 1894-1912; Clarendon Press: Oxford, UK, 1978.

11. Milonni, P.W. The Quantum Vacuum; Academic Press: San Diego, CA, USA, 1994; p. 522.

12. Kragh, H. Preludes to dark energy: Zero-point energy and vacuum speculations. Arch. Hist. Exact Sci. 2012, 66, 199-240. [CrossRef]

13. Power, E.A. Introductory Quantum Electrodynamics; American Elsevier Publishing Company: New York, NY, USA, 1965.

14. Wang, S.C. Die gegenseitige Einwirkung zweier Wasserstoffatome. Phys. Z. 1927, 28, 663-666.

15. Von Eisenschitz, R.; London, F. Uber das Verhaltnis der van der Waalsschen Krafte zu den homoopolaren Bindungskraften. Z. Phys. 1930, 60, 491-527. [CrossRef]

16. Pais, A. Imward Bound; Oxford University Press: Oxford, UK, 1986.

17. London, F. The general theory of molecular forces. Trans. Faraday Soc. 1937, 33, 8-26, [CrossRef]

18. Margenau, H. The role of quadrupole forces in van der Waals attractions. Phys. Rev. 1931, 38, 747-756. [CrossRef]

19. Margenau, H. Quadrupole Contributions to London's dispersion forces. J. Chem. Phys. 1938, 6, 896-899. [CrossRef]

20. Verwey, E.J.W.; Overbeek, J.T.G. Theory of the Stability of Lyophobic Colloids; Elsevier Publishing Company, Inc.: New York, NY, USA, 1948; pp. 1-108.

21. Casimir, H.B.G. Some remarks on the history of the so-called Casimir effect. In The Casimir Effect 50 Years Later, Proceedings of the Fourth Workshop on Quantum Field Theory Under the Influence of External Conditions, Leipzig, Germany, 14-18 September 1998; Bordag, M., Ed.; World Scientific Publishing Co., Pte. Ltd.: Singapore, 1999; pp. 3-9. 
22. Pinto, F. Casimir forces: Fundamental theory, computation, and nanodevices applications. In Quantum Nano-Photonics, NATO Science for Peace and Security Series B: Physics and Biophysics (Erice, Sicily, Italy); Di Bartolo, B., Ed.; Springer Nature B.V.: Berlin/Heidelberg, Germany, 2018; pp. 149-180.

23. Casimir, H.B.G.; Polder, D. Influence of retardation on the London-van-der-Waals forces. Nature 1946, 158, 787-788. [CrossRef]

24. Casimir, H.B.G.; Polder, D. The influence of retardation on the London-van der Waals forces. Phys. Rev. 1948, 73, 360-372. [CrossRef]

25. Casimir, H.B.G. On the history of the so-called Casimir effect. In Comments on Atomic and Molecular Physics, Comments on Modern Physics. Part D (Special Issue: Casimir Forces); Babb, J.F., Milonni, P.W., Spruch, L., Eds.; Gordon and Breach Science Publishers: Kuala Lumpur, Malaysia, 2000; Volume 1, pp. 175-177.

26. Casimir, H.B.G. Van der Waals forces and zero point energy. In Physics of Strong Fields; Greiner, W., Ed.; Springer US: New York, NY, USA, 1987; pp. 957-964.

27. Casimir, H.B.G. Van der Waals forces and zero point energy. In Essays in Honour of Victor Frederick Weisskopf (Physics and Society); Springer: New York, NY, USA, 1998; pp. 53-66.

28. Einstein, A. Folgerungen aus den Capillaritätserscheinungen. Ann. Der Phys. 1901, 4, 513-523, [CrossRef]

29. Einstein, A. Conclusions drawn from the phenomena of capillarity. In The Collected Papers of Albert Einstein, Vol. 2 (The Swiss Years, Scientific Writings, 1900-1909); Princeton University Press: Princeton, NJ, USA, 1901; pp.1-11.

30. de Boer, J.H. The influence of van der Waals forces and primary bonds on binding energy, strength and special reference to some artificial resins. Trans. Faraday Soc. 1936, 32, 10-37. [CrossRef]

31. Hamaker, H. The London-van der Waals attraction between spherical particles. Physica 1937, 4, 1058-1072, [CrossRef]

32. Rowlinson, J.S. Cohesion-A Scientific History of Intermolecular Forces; Cambridge University Press: Cambridge, UK, $2002 ;$ pp. 1-333.

33. Axilrod, B.M.; Teller, E. Interaction of the van der Waals Type Between Three Atoms. J. Chem. Phys. 1943, 11, 299-300. [CrossRef]

34. Lifshitz, E.M. The theory of molecular attractive forces between solids. Sov. Phys. JETP 1956, 2, $73-83$.

35. Dzyaloshinskii, I.; Lifshitz, E.M.; Pitaevskii, L.P. The general theory of van der Waals forces. Adv. Phys. 1961, 10, 165-209. [CrossRef]

36. Bordag, M.; Klimchitskaya, G.L.; Mohideen, U.; Mostepanenko, V.M. Advances in the Casimir Effect; Oxford University Press: Oxford, UK, 2009.

37. Klimchitskaya, G.L.; Mohideen, U.; Mostepanenko, V.M. The Casimir force between real materials: Experiment and theory. Rev. Mod. Phys. 2009, 81, 1827-1885, [CrossRef]

38. Dalvit, D.A.R.; Milonni, P.W.; Roberts, D.; da Rosa, F. Casimir Physics; Springer Lecture Notes in Physics 834; Springer: Berlin/Heidelberg, Germany, 2011.

39. Lamoreaux, S.K. The Casimir Force and Related Effects: The Status of the Finite Temperature Correction and Limits on New Long-Range Forces. Annu. Rev. Nucl. Part. Sci. 2012, 62, 37-56, [CrossRef]

40. Schwinger, J.; DeRaad, L.L.; Milton, K.A. Casimir effect in dielectrics. Ann. Phys. 1978, 23, 1-23. [CrossRef]

41. Saunders, S. Is the Zero-Point Energy Real? In Ontological Aspects of Quantum Field Theory; Kuhlmann, M., Lyre, H., Wayne, A., Eds.; World Scientific: Hackensack, NJ, USA, 2002; Chapter 16, pp. 313-343.

42. Langbein, D. Theory of van der Waals Attraction; Springer: Berlin/Heidelberg, Germany, 1974; pp. 1-144.

43. Copernicus, N. On the Revolutions of the Heavenly Spheres; Great Books of the Western World; William Benton: Chicago, IL, USA, 1952; Volume 16,.

44. Copernico, N. De Revolutionibus Orbium Caelestium—La Costituzione Generale Dell'universo-A Cura di Alexandre Koyré; Giulio Einaudi Editore s.p.a.: Torino, Italy, 1975.

45. Lamoreaux, S.K. Casimir forces: Still surprising after 60 years. Phys. Today 2007, 60, 40-45. [CrossRef]

46. Karplus, M.; Porter, R.N. Atoms \& Molecules; The Benjamin/Cummings Publishing Company: Menlo Park, CA, USA, 1970.

47. Kleppner, D. With apologies to Casimir. Phys. Today 1990, 43, 9-11. [CrossRef]

48. Mahanty, J.; Ninham, B.W. Dispersion Forces; Academic Press: London, UK, 1976.

49. McLachlan, A.D. Retarded Dispersion Forces Between Molecules. Proc. Roy. Soc. (Lond.) Ser. A 1963, $271,387-401$.

50. Margenau, H.; Kestner, N.R. Theory of Intermolecular Forces, 2nd ed.; Pergamon Press: Oxford, UK, 1971; pp. 1-400.

51. Marshall, T.W. Random Electrodynamics. Proc. R. Soc. Math. Phys. Eng. Sci. 1963, 276, 475-491. [CrossRef]

52. Marshall, T.W. Statistical electrodynamics. Proc. Camb. Phil. Soc. 1965, 61, 537-546. [CrossRef]

53. Boyer, T.H. Recalculations of long-range van der Waals potentials. Phys. Rev. 1969, 180, 19-24. [CrossRef]

54. Boyer, T.H. Van der Waals forces and zero-point energy for dielectric and permeable materials. Phys. Rev. A 1974, 9, 2078-2084. [CrossRef]

55. Hushwater, V. Repulsive Casimir force as a result of vacuum radiation pressure. Am. J. Phys. 1997, 65, 381-384, [CrossRef]

56. Spruch, L.; Kelsey, J. Vacuum fluctuation and retardation effects on long-range potentials. Phys. Rev. A 1978, 18, 845-852. [CrossRef]

57. Spruch, L. Retarded, or Casimir, Long-Range Potentials. Phys. Today 1986, 39, 37-45. [CrossRef]

58. Boyer, T.H. Blackbody Radiation and the Scaling Symmetry of Relativistic Classical Electron Theory with Classical Electromagnetic Zero-Point Radiation. Found. Phys. 2010, 40, 1096-1098. [CrossRef]

59. Camparo, J. Semiclassical description of radiative decay in a colored vacuum. Phys. Rev. A 2001, 65, 13815. [CrossRef] 
60. Pinto, F. Gravitational Casimir effect, the Lifshitz theory, and the existence of gravitons. Class. Quantum Grav. 2016, 33, 237001. [CrossRef]

61. González, A.E. On Casimir pressure, the Lorentz force and black body radiation. Physica 1985, 131A, 228-236. [CrossRef]

62. Milonni, P.W.; Cook, R.J.; Goggin, M.E. Radiation pressure from the vacuum: Physical interpretation of the Casimir force. Phys. Rev. A 1988, 38, 1621-1623. [CrossRef]

63. Barton, G. On the fluctuations of the Casimir force. J. Phys. A Math. Gen. 1991, 24, 991-1005. [CrossRef]

64. Susbilla, R.T. Casimir Acoustics. Ph.D. Thesis, Naval Postgraduate School, Monterey, CA, USA, 1996.

65. Holmes, C.D. Acoustic Casimir Effect. Ph.D. Thesis, Naval Postgraduate School, Monterey, CA, USA, 1997.

66. Larraza, A.; Denardo, B. An acoustic Casimir effect. Phys. Lett. A 1998, 248, 151-155. [CrossRef]

67. Larraza, A.; Holmes, C.D.; Susbilla, R.T.; Denardo, B.C. The force between two parallel rigid plates due to the radiation pressure of broadband noise: An acoustic Casimir effect. J. Acoust. Soc. Am. 1998, 103, 2267-2272. [CrossRef]

68. Ford, L.H. Spectrum of the Casimir effect and the Lifshitz theory. Phys. Rev. A 1993, 48, 2962-2967. [CrossRef] [PubMed]

69. Esquivel-Sirvent, R.; Reyes, L.I. Pull-in control in microswitches using acoustic Casimir forces. EPL 2008, 84, 48002. [CrossRef]

70. Serry, F.M.; Walliser, D.; Maclay, G. The anharmonic Casimir oscillator (ACO)-the Casimir effect in a model microelectromechanical system. J. Microelectromech. Syst. 1995, 4, 193-205. [CrossRef]

71. Boersma, S.L. A maritime analogy of the Casimir effect. Am. J. Phys. 1996, 64, 539-541. [CrossRef]

72. Denardo, B.C.; Puda, J.J.; Larraza, A. A water wave analog of the Casimir effect. Am. J. Phys. 2009, 77, 1095. [CrossRef]

73. Ball, P. Popular physics myth is all at sea. Nature 2006, 2006-2008. [CrossRef]

74. Fisher, D.J. Maritime Casimir effect. Am. J. Phys. 1996, 64, 1228. [CrossRef]

75. Brügger, G.; Froufe-Pérez, L.S.; Scheffold, F.; Sáenz, J.J. Controlling dispersion forces between small particles with artificially created random light fields. Nat. Commun. 2015, 6, 7460. [CrossRef]

76. Holzmann, D.; Ritsch, H. Tailored long range forces on polarizable particles by collective scattering of broadband radiation. New J. Phys. 2016, 18, 103041. [CrossRef]

77. Shi, Y.Z.; Xiong, S.; Zhang, Y.; Chin, L.K.; Chen, Y.Y.; Zhang, J.B.; Zhang, T.H.; Ser, W.; Larrson, A.; Lim, S.H.; et al. Sculpting nanoparticle dynamics for single- bacteria-level screening and direct binding-efficiency measurement. Nat. Commun. 2018, 9, 815 [CrossRef]

78. Thirunamachandran, T. Intermolecular interactions in the presence of an intense radiation field. Mol. Phys. 1980, 40, 393-399. [CrossRef]

79. Craig, D.P.; Thirunamachandran, T. Molecular Quantum Electrodynamics; Dover Publications, Inc.: Mineola, NY, USA, 1998.

80. O'Dell, D.; Giovanazzi, S.; Kurizki, G.; Akulin, V.M. Bose-Einstein condensates with 1/r interatomic attraction: Electromagnetically induced "gravity". Phys. Rev. Lett. 2000, 84, 5687-5690. [CrossRef] [PubMed]

81. Luis-Hita, J.; Marqués, M.I.; Delgado-Buscalioni, R.; de Sousa, N.; Froufe-Pérez, L.S.; Scheffold, F.; Sáenz, J.J. Light Induced Inverse-Square Law Interactions between Nanoparticles: "Mock Gravity" at the Nanoscale. Phys. Rev. Lett. $2019,123,143201$. [CrossRef] [PubMed]

82. Burns, M.M.; Fournier, J.M.; Golovchenko, J.A. Optical binding. Phys. Rev. Lett. 1989, 63, 1233-1236. [CrossRef] [PubMed]

83. DeWitt, B.S. The Casimir effect in field theory. In Physics in the Making; Sarlemijn, A., Sparnaay, M.J., Eds.; Elsevier Science Publishers: Amsterdam, The Netherlands, 1989; Chapter 9B, pp. 247-272.

84. Lamoreaux, S.K. Demonstration of the Casimir Force in the 0.6 to $6 \mu \mathrm{m}$ Range. Phys. Rev. Lett. 1997, 78, 5-8. [CrossRef]

85. Lamoreaux, S.K. The Casimir force: background, experiments, and applications. Rep. Prog. Phys. 2004, 68, 201-236. [CrossRef]

86. Wright, A. Milestone 6. QED. Nat. Mater. 2010, 9, pS9. [CrossRef]

87. Feynman, R.P. The development of the space-time view of quantum-electrodynamics. Science 1966, 153, 699-708. [CrossRef]

88. Pinto, F. Resolution of a paradox in classical electrodynamics. Phys. Rev. D 2006, 73, 104020, [CrossRef]

89. Fermi, E. Sull'elettrostatica di un campo gravitazionale uniforme e sul peso delle masse elettromagnetiche. Il Nuovo Cimento 1921, 22, 176-188. [CrossRef]

90. Gorelik, G.E. The First Steps of Quantum Gravity and the Planck Values. In Studies in the History of General Relativity; Eisenstaedt, J., Kox, A., Eds.; Eistenin Studies; Birkhäuser: Basel, Switzerland, 1992; Volume 3, p. 367.

91. Stachel, J. The Early History of Quantum Gravity (1916-1940). In Black Holes, Gravitational Radiation and the Universe; Iyer, B.R., Bhawal, B., Eds.; Fundamental Theories of Physics; Springer Science: Dordrecht, the Netherlands, 1999; Volume 100, Chapter 31, pp. 525-534.

92. Kiefer, C. Quantum Gravity; Oxford University Press: Oxford, UK, 2007.

93. Rosenfeld, L. Zur Quantelung der Wellenfelder. Ann. Phys. 1930, 397, 113-152. [CrossRef]

94. Bronstein, M. Quantentheorie schwacher Gravitationsfelder. Phys. Z. Sowjetunion 1936, 9, 140-157.

95. Deser, S.; Starobinsky, A. Editorial note to: Matvei P. Bronstein, Quantum theory of weak gravitational field. Gen. Relativ. Gravit. 2012, 44, 263-265. [CrossRef]

96. Bronstein, M. Republication of: Quantum theory of weak gravitational fields. Gen. Relativ. Gravit. 2012, 44, 267-283. [CrossRef]

97. Gorelik, G.E.; Frenkel, V.Y. Matvei Petrovich Bronstein and Soviet Theoretical Physics in the Thirties, 1st ed.; Springer: Basel, Switzerland, 1994.

98. Planck, M. Über irreversible Strahlungsvorgänge. Sitzungsberichte Königlich-Preußischen Akad. Wiss. 1899, 5, 440-480.

99. DeWitt, C.M.; DeWitt, B.S. Falling charges. Physics 1964, 1, 3-20. [CrossRef] 
100. Berends, F.A.; Gastmans, R. Quantum electrodynamical corrections to graviton-matter vertices. Ann. Phys. 1976, 98, 225-236. [CrossRef]

101. Milton, K.A. Quantum-electrodynamic corrections to the gravitational interaction of the electron. Phys. Rev. D 1977, 15, 538-540. [CrossRef]

102. Barker, B.M.; O'Connell, R.F. Post-Newtonian two-body and n-body problems with electric charge in general relativity. J. Math Phys. 1977, 18, 1818-1824. [CrossRef]

103. Gupta, S.N.; Radford, S.F. Quantum field-theoretical electromagnetic and gravitational two-particle potentials. Phys. Rev. D 1980, 21, 2213-2225. [CrossRef]

104. Butt, M.S. Leading quantum gravitational corrections to QED. Phys. Rev. D 2006, 74, 125007. [CrossRef]

105. Holstein, B.R. Graviton physics. Am. J. Phys. 2006, 74, 1002-1011. [CrossRef]

106. Holstein, B.R.; Ross, A. Long Distance Effects in Mixed Electromagnetic-Gravitational Scattering. arXiv 2008, arXiv:0802.0717.

107. Holstein, B.R. Analytical On-shell Calculation of Low Energy Higher Order Scattering. J. Phys. G Nucl. Part. Phys. $2017,44,1$ LT01. [CrossRef]

108. Feinberg, G.; Sucher, J.; Au, C.K. The dispersion theory of dispersion forces. Phys. Rep. 1989, 180, 83-157. [CrossRef]

109. Spruch, L. An overview of long-range Casimir interactions. In Long-Range Casimir Forces; Levin, F.S., Micha, D.A., Eds.; Springer Science + Business Media: New York, NY, USA, 1993; Chapter 1, pp. 1-71.

110. Dowling, J.P. Retarded potentials (Letter). Phys. Today 1991, 39, 13-15. [CrossRef]

111. Barut, A.O. Electromagnetic Interactions beyond Quantum Electrodynamics. In Foundations of Radiation Theory and Quantum Electrodynamics; Barut, A.O., Ed.; Springer Science + Business Media: Berlin/Heidelberg, Germany, 1980; Chapter 14, pp. 165-172.

112. Barut, A.O. Quantum-electrodynamics based on self-energy. Phys. Scr. 1988, T21, 18-21. [CrossRef]

113. Barut, A.O.; Van Huele, J.F. Quantum electrodynamics based on self-energy: Lamb shift and spontaneous emission without field quantization. Phys. Rev. A 1985, 32, 3187-3195. [CrossRef]

114. Barut, A.O.; Dowling, J.P. Quantum electrodynamics based on self-energy, without second quantization: The Lamb shift and long-range Casimir-Polder van der Waals forces near boundaries. Phys. Rev. A 1987, 36, 2550-2556. [CrossRef]

115. Barut, A.O.; Dowling, J.P. Self-field quantum electrodynamics: The two-level atom. Phys. Rev. A 1990, 41, 2284-2294. [CrossRef]

116. Appelquist, T.; Chodos, A. Quantum effects in Kaluza-Klein theories. Phys. Rev. Lett. 1983, 50, 141-145. [CrossRef]

117. Appelquist, T.; Chodos, A. Quantum dynamics of Kaluza-Klein theories. Phys. Rev. D 1983, 28, 772-784. [CrossRef]

118. Abe, O. Casimir Energy in Quantum Gravity in R1 times T3 Space-Time. Prog. Theor. Phys. 1984, 72, 1225-1232. [CrossRef]

119. Panella, O.; Widom, A. Casimir effects in gravitational interactions. Phys. Rev. D 1994, 49, 917-922. [CrossRef]

120. Panella, O.; Widom, A.; Srivastava, Y.N. Casimir effects for charged particles. Phys. Rev. B 1990, 42, 9790-9793. [CrossRef]

121. Torr, D.G.; Li, N. Gravitoelectric-electric coupling via superconductivity. Found. Phys. Lett. 1993, 6, 371-383. [CrossRef]

122. Woods, R.C. Manipulation of gravitational waves for communications applications using superconductors. Phys. C 2005, 433, 101-107. [CrossRef]

123. Garcia-Cuadrado, G. Towards a New Era in Gravitational Wave Detection: High Frequency Gravitational Wave Research. In Proceedings of the Space, Propulsion \& Energy Sciences International Forum SPESIF-2009, Huntsville, AL, USA, 24-27 February 2009; Volume 1103, pp. 553-563, [CrossRef]

124. Woods, R.C. Interactions between supeconductors and high-frequency gravitational waves. In Gravity-Superconductors Interactions: Theory and Experiment; Modanese, G., Robertson, G.A., Eds.; Bentham: Sharjah, UAE, 2012; pp. $23-57$.

125. Minter, S.J.; Wegter-McNelly, K.; Chiao, R.Y. Do mirrors for gravitational waves exist? Phys. E Low-Dimens. Syst. Nanostruct. 2010, 42, 234-255. [CrossRef]

126. Sakharov, A.D. Vacuum Quantum Fluctuations in Curved Space and the Theory of Gravitation. Gen. Relativ. Gravit. 2000, 32, 365-367. [CrossRef]

127. Visser, M. Sakharov's induced gravity: A modern perspective. Mod. Phys. Lett. A 2002, 17, 977-991. [CrossRef]

128. Belgiorno, F.; Liberati, S. Black hole thermodynamics, Casimir effect and Induced Gravity. Gen. Relativ. Gravit. 1997, $29,1181-1194$. [CrossRef]

129. Quach, J.Q. Gravitational Casimir Effect. Phys. Rev. Lett. 2015, 114, 81104. [CrossRef] [PubMed]

130. Quach, J.Q. Erratum: Gravitational Casimir Effect. Phys. Rev. Lett. 2017, 118, 139901. [CrossRef] [PubMed]

131. Ford, L.H.; Hertzberg, M.P.; Karouby, J. Quantum Gravitational Force Between Polarizable Objects. Phys. Rev. Lett. 2016, 116, 151301. [CrossRef]

132. Wu, P.; Hu, J.; Yu, H. Quantum correction to classical gravitational interaction between two polarizable objects. Phys. Lett. B 2016, 763, 40-44. [CrossRef]

133. Hu, J.; Yu, H. Gravitational Casimir-Polder effect. Phys. Lett. B 2017, 767, 16-19. [CrossRef]

134. Holstein, B.R. Connecting Compton and Gravitational Compton Scattering. EPJ Web Conf. 2017, 134, 1003. [CrossRef]

135. Rini, M. Synopsis: A Casimir Effect Caused by Gravity. Physics 2015, 8, s23.

136. Becker, A. Ultra-cold mirrors could reveal gravity's quantum side. New Sci. 2015. [CrossRef]

137. Hossenfelder, S. Can we prove the quantization of gravity with the Casimir effect? Probably not. arXiv 2015, arXiv:1502.07429.

138. Pinto, F. If detected, would hypothetical gravitational Casimir effects prove gravity quantization? In Proceedings of the Fourteenth Marcel Grossman Meeting on General Relativity, Rome, Italy, $12-18$ July 2015; Bianchi, M., Jantzen, R.T., Ruffini, R., Eds.; World Scientific: Singapore, 2017; pp. 3966-3973. [CrossRef] 
139. Abbott, B.P. Observation of Gravitational Waves from a Binary Black Hole Merger. Phys. Rev. Lett. 2016, 116, 61102. [CrossRef] [PubMed]

140. Griffiths, D.J.; Ho, E. Classical Casimir effect for beads on a string. Am. J. Phys. 2001, 69, 1173-1176. [CrossRef]

141. Boyer, T.H. Casimir forces and boundary conditions in one dimension: Attraction, repulsion, Planck spectrum, and entropy. Am. J. Phys. 2003, 71, 990-998. [CrossRef]

142. Dev, P.S.B.; Mazumdar, A. Probing the scale of new physics by Advanced LIGO/VIRGO. Phys. Rev. D 2016, 93, 104001. [CrossRef]

143. Ross, D.K.; Moreau, W. Stochastic Gravity. Gen. Relativ. Gravit. 1995, 27, 845-858. [CrossRef]

144. Allen, B. The stochastic gravity-wave background: Sources and detection. In Proceedings of the Les Houches School on Astrophysical Sources of Gravitational Waves, Les Houches, France, 26 September-6 October 1995; pp. 373-417.

145. Lasky, P.D.; Mingarelli, C.M.F.; Smith, T.L.; Giblin, J.T.; Thrane, E.; Reardon, D.J.; Caldwell, R.; Bailes, M.; Bhat, N.D.R.; Burke-spolaor, S.; et al. Gravitational-Wave Cosmology across 29 Decades in Frequency. Phys. Rev. X 2016, 6, 11035. [CrossRef]

146. Quiñones, D.A.; Oniga, T.; Varcoe, B.T.H.; Wang, C.H. Quantum principle of sensing gravitational waves: From the zero-point fluctuations to the cosmological stochastic background of spacetime. Phys. Rev. D 2017, 96, 44018. [CrossRef]

147. Rugh, S.E.; Zinkernagel, H.; Cao, T.Y. The Casimir Effect and the Interpretation of the Vacuum. Stud. Hist. Philos. Sci. Part Stud. Hist. Philos. Mod. Phys. 1999, 30, 111-139. [CrossRef]

148. Pinto, F. Improved finite-difference computation of the van der Waals force: One-dimensional case. Phys. Rev. A 2009, 80, 42113. [CrossRef]

149. Cohen-Tannoudji, C.; Diu, B.; Laloe, F. Quantum Mechanics (Two Volumes); John Wiley \& Sons: New York, NY, USA, 1977.

150. Power, E.A.; Thirunamachandran, T. Dispersion interactions between atoms involving electric quadrupole polarizabilities. Phys. Rev. A 1996, 53, 1567-1575. [CrossRef]

151. Davydov, A.S. Quantum Mechanics; Pergamon Press: Oxford, UK, 1965; Volume 1.

152. Jackson, J.D. Classical Electrodynamics (Second Edition); John Wiley \& Sons: New York, NY, USA, 1975.

153. Pauling, L.; Beach, J.Y. The van der Waals interaction of hydrogen atoms. Phys. Rev. 1935, 47, 686-692. [CrossRef]

154. Pauling, L.; Wilson, B.E.J. Introduction to Quantum Mechanics; Dover Publications, Inc.: Mineola, NY, USA, $1985 ;$ pp. 1-468.

155. Raab, R.E.; de Lange, O.L. Multipole Theory in Electromagnetism; Oxford University Press: Oxford, UK, 2005.

156. Salam, A.; Thirunamachandran, T. A new generalization of the Casimir-Polder potential to higher electric multipole polarizabilities. J. Chem. Phys. 1996, 104, 5094-5099. [CrossRef]

157. Salam, A. Non-Relativistic QED Theory of the van der Waals Dispersion Interaction; Springer: Cham, Switzerland, 2016.

158. Golub, G.H.; Van Loan, C.F. Matrix Computations, 3rd ed.; The Johns Hopkins University Press: Baltimore, MD, USA; London, UK, 1996.

159. Jenkins, J.K.; Salam, A.; Thirunamachandran, T. Retarded dispersion interaction energies between chiral molecules. Phys. Rev. A 1994, 50, 4767-4777. [CrossRef] [PubMed]

160. Szekeres, P. Linearized gravitation theory in macroscopic media. Ann. Phys. 1971, 64, 599-630. [CrossRef]

161. Szekeres, P. Gravitational fields in matter. In Relativity and Gravitation; Kuper, C.G., Peres, A., Eds.; Gordon and Breach Science Publishers: New York, NY, USA, 1971; pp. 305-308.

162. Pinto, F. Rydberg atoms as gravitational-wave antennas. Gen. Relativ. Gravit. 1995, 27, 9-14, [CrossRef]

163. Pinto, F. Gravitational-wave response of parametric amplifiers driven by radiation-induced dispersion force modulation. In Proceedings of the Fourteenth Marcel Grossmann Meeting on General Relativity, Rome, Italy, 12-18 July 2015; Bianchi, M., Jantzen, R.T., Ruffini, R., Eds.; World Scientific: Singapore, 2017; pp. 3175-3182, [CrossRef]

164. Tourrenc, P.; Grossiord, J.L. Modification du spectre de l'hydrogène atomique sous l'influence de champs de gravitation. Il Nuovo Cimento 1976, 32B, 163-176. [CrossRef]

165. Parker, L. One-electron atom in curved space-time. Phys. Rev. Lett. 1980, 44, 1559-1562. [CrossRef]

166. Pinto, F. Rydberg atoms in curved space-time. Phys. Rev. Lett. 1993, 70, 3839-3843. [CrossRef]

167. Misner, C.W.; Thorne, K.S.; Wheeler, J.A. Gravitation; W. H. Freeman and Company: San Francisco, CA, USA, $1970 ;$ pp. 1-1304.

168. Schutz, B.F. A First Course in General Relativity; Cambridge University Press: Cambridge, UK, 2009; pp. 1-393.

169. Price, R.H.; Belcher, J.W.; Nichols, D.A. Comparison of electromagnetic and gravitational radiation: What we can learn about each from the other. Am. J. Phys. 2013, 81, 575-584. [CrossRef]

170. McLachlan, A.D.; Gregory, R.D.; Ball, M.A. Molecular interactions by the time-dependent Hartree method. Mol. Phys. 1964, 7, 119-129. [CrossRef]

171. McLachlan, A.D. Retarded dispersion forces in dielectrics at finite temperatures. Proc. R. Soc. Lond. Ser. Math. Phys. Sci. 1963, 274, 80-90.

172. Thirunamachandran, T. Vacuum fluctuations and intermolecular interactions. Phys. Scr. 1988, T21, 123-128. [CrossRef]

173. Power, E.A.; Thirunamachandran, T. A new insight into the mechanism of intermolecular forces. Chem. Phys. 1993, 171, 1-7. [CrossRef]

174. Renne, M.J. Retarded van der Waals interaction in a system of harmonic oscillators. Physica 1971, 53, 193-209. [CrossRef]

175. Renne, M.J. Microscopic theory of retarded van der Waals forces between macroscopic dielectric bodies. Physica 1971, 56, $125-137$. [CrossRef]

176. Goldstein, H.; Poole, C.; Safko, J. Classical Mechanics, 3rd ed.; Addison-Wesley: San Francisco, CA, USA, $2002 ;$ pp. 1-647.

177. Hinshelwood, C.N. The Structure of Physical Chemistry; Oxford University Press: London, UK, 1951. 
178. Panofski, W.K.H.; Phillips, M. Classical Electricity and Magnetism, 2nd ed.; Addison-Wesley Publ. Co., Inc.: Reading, MA, USA, 1962; pp. 1-503.

179. Shadowitz, A. The Electromagnetic Field; Dover Publications, Inc.: New York, NY, USA, 1975.

180. Passante, R. Dispersion Interactions between Neutral Atoms and the Quantum Electrodynamical Vacuum. Symmetry 2018, 10, 735. [CrossRef]

181. Power, E.A.; Thirunamachandran, T. Dispersion forces between molecules with one or both molecules excited. Phys. Rev. A 1995, 51, 3660-3666. [CrossRef]

182. Zangwill, A. Modern Electrodynamics; Cambridge University Press: Cambridge, UK, 2013; pp. 1-977.

183. Good, R.H.; Nelson, T.J. Classical Theory of Electric and Magnetic Fields; Academic Press: New York, NY, USA, 1971.

184. Weber, J. General Relativity and Gravitational Waves; Interscience Publishers, Inc.: New York, NY, USA, 1961.

185. Bimonte, G.; Calloni, E.; Esposito, G.; Rosa, L. Energy-momentum tensor for a Casimir apparatus in a weak gravitational field. Phys. Rev. D 2006, 74, 085011. [CrossRef]

186. Norte, R.A.; Forsch, M.; Wallucks, A.; Marinković, I.; Gröblacher, S. Platform for Measurements of the Casimir Force between Two Superconductors. Phys. Rev. Lett. 2018, 121, 30405. [CrossRef]

187. Pinto, F. A trapped dipolar BEC interferometry test of $\mathrm{E}=\mathrm{mc}^{2}$. Int. J. Mod. Phys. D 2006, 15, 2235-2240. [CrossRef]

188. Bimonte, G.; Born, D.; Calloni, E.; Esposito, G.; Il'ichev, E.; Rosa, L.; Scaldaferri, O.; Tafuri, F.; Vaglio, R.; Hübner, U. The Aladin2 experiment: status and perspectives. J. Phys. Math. Gen. 2006, 39, 6153-6159. [CrossRef]

189. Allocca, A.; Bimonte, G.; Born, D.; Calloni, E.; Esposito, G.; Huebner, U.; Il, E.; Francesco, R. Results of Measuring the Influence of Casimir Energy on Superconducting Phase Transitions. J. Supercond. Nov. Magn. 2012, 25, 2557-2565. [CrossRef]

190. Ignat'ev, Y.G.; Zakharov, A.V. The reflection of gravitational waves from compact stars. Phys. Lett. 1978, 66A, 3-4. [CrossRef]

191. Li, N.; Torr, G. Gravitational effects on the magnetic attenuation of superconductors. Phys. Rev. B 1992, 46, 5489-5495. [CrossRef]

192. Klimchitskaya, G.L.; Mostepanenko, V.M. Recent measurements of the Casimir force: Comparison between experiment and theory. Mod. Phys. Lett. A 2020, 35, 2040007. [CrossRef]

193. Pinto, F. Efimov physics in curved spacetime: Field fluctuations and exotic matter. Int. J. Mod. Phys. D 2018, $27,1847001$. [CrossRef]

194. Pinto, F. Signatures of minimal length from Casimir-Polder forces with neutrons. Int. J. Mod. Phys. D 2020, 2043026. [CrossRef]

195. Pinto, F. The history of technology transfer of the Casimir effect and van der Waals forces: From exotic, weak, and undesirable to enabling, emerging, and irresistible. In Proceedings of the Atti del XXXIX Convegno Annuale Della SISFA, Pisa, Italy, 9-12 September 2019; La Rana, A., Possi, P., Eds.; Pisa University Press: Pisa, Italy, 2020; pp. 281-287. [CrossRef]

196. Post, H.R. The Problem of Atomism. Br. J. Philos. Sci. 1975, 26, 19-26. [CrossRef]

197. Wheeler, J.A. From relativity to mutability. In The Physicist's Conception of Nature; Mehra, J., Ed.; D. Reidel Publishing Company: Dordrecht, The Netherlands, 1973; Chapter 9, pp. 202-247.

198. Pinto, F. Dispersion force engineering and next-generation spacecraft: Case studies in a nanoscale emerging enabling generalpurpose technology. Materials Today. In Proceedings of the 17th International Conference on Nanosciences \& Nanotechnologies (NN20), Thessaloniki, Greece, 7-10 July 2020.

199. Hawkes, E.W.; Eason, E.V.; Christensen, D.L.; Cutkosky, M.R. Human climbing with efficiently scaled gecko-inspired dry adhesives. J. R. Soc. Interface 2015, 12, 20140675, [CrossRef]

200. Pinto, F. Mixed electric-gravitational Casimir-Polder potentials. In Proceedings of the 4th Symposium on the Casimir Effect, Saint Petersburg, Russia, 23-29 June 2019. 